\title{
Stability of Flows in Fluidized Beds
}

\author{
Final Report
}

Work Performed Under Contract No.: DE-AC21-89MC26302

\author{
For \\ U.S. Department of Energy \\ Office of Fossil Energy \\ Morgantown Energy Technology Center \\ P.O. Box 880 \\ Morgantown, West Virginia 26507-0880 \\ Ly \\ Viscoustech \\ 4693 Magn us Drive \\ Allison Park, Pennsylvania 15101-2511
}

April 1993 


\begin{abstract}
We study the linearized stability of the state of uniform fluidization, within the context of the theory of mixtures. The mixture is assumed to be made up of a classical linearly viscous fluid mixed with solid particles. In marked departure from most of the previous studies, we model the solid as a granular material and assume a constitutive relation that stems from classicul motions in continuum mechanics. The linearized stability analysis of the state of uniform fluidization, in general, leads to an eighth order equation for the characteristic whose root implies instability, when positive. We find that the characteristic equation can be factored and one of the factors is precisely the equation that governs the stability when we use a simplified analysis akin to that of the earlier study of Anderson and Jackson.
\end{abstract}




\section{TABLE OF CONTENTS}

LIST OF TABLES $\ldots \ldots \ldots \ldots \ldots \ldots \ldots \ldots \ldots \ldots \ldots \ldots \ldots \ldots \ldots \ldots, v$

1.0 INTRODUCTION $\ldots \ldots \ldots \ldots \ldots \ldots \ldots \ldots \ldots \ldots \ldots \ldots \ldots \ldots \ldots$

1.1 Fluidized Beds $\ldots \ldots \ldots \ldots \ldots \ldots \ldots \ldots \ldots \ldots \ldots \ldots \ldots \ldots$

1.2 Hydrodynamic Stability $\ldots \ldots \ldots \ldots \ldots \ldots \ldots \ldots \ldots \ldots \ldots \ldots$

2.0 PRELIMINARIES AND BALANCE LAWS ................ 11

2.1 Conservation of Mass $\ldots \ldots \ldots \ldots \ldots \ldots \ldots \ldots \ldots \ldots, 12$

2.2 Conservation of Linear Momentum $\ldots \ldots \ldots \ldots \ldots \ldots \ldots .14$

3.0 CONSTITUTIVE MODELING $\ldots \ldots \ldots \ldots \ldots \ldots \ldots \ldots \ldots \ldots \ldots \ldots$

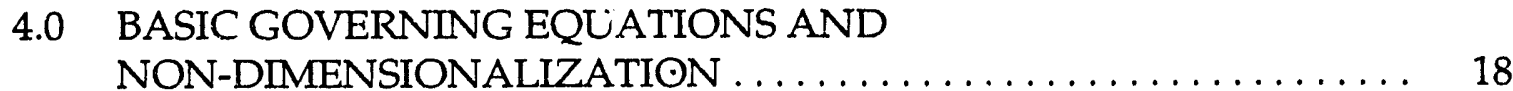

4.1 Conservation of Mass for the Solid Constituent ........... 19

4.2 Conservation of Linear Momentum for the Solid Constituent ... 19

4.3 Conservation of Mass for the Fluid Constituent ........... 20

4.4 Conservation of Linear Momentum for the Fluid Constituent ... 20

5.0 STATE OF UNIFORM FLUIDIZATION $\ldots \ldots \ldots \ldots \ldots \ldots \ldots \ldots . \ldots \ldots$

6.0 PERTURBANCES TO THE STATE OF UNIFORM FLUIDIZATION .. 22

6.1 Equations of Order $\varepsilon^{0} \ldots \ldots \ldots \ldots \ldots \ldots \ldots \ldots \ldots \ldots \ldots \ldots, 22$

6.2 Equations of $\operatorname{Order} \varepsilon^{1} \ldots \ldots \ldots \ldots \ldots \ldots \ldots \ldots \ldots \ldots \ldots \ldots \ldots, 23$

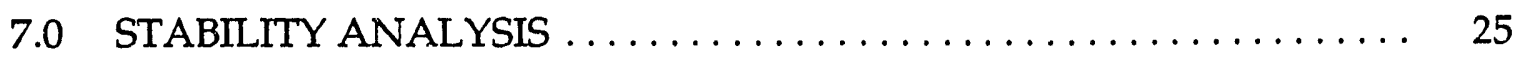

8.0 A SIMPLIFIED STABILITY ANALYSIS . . . . . . . . . . . . 33

9.0 STABILIZING ROLE OF THE SPHERICAL PART OF THE STRESS

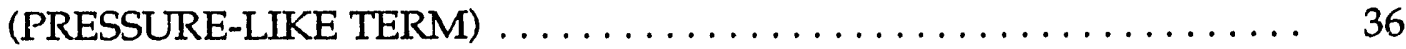

10.0 RESULTS AND CONCLUSION $\ldots \ldots \ldots \ldots \ldots \ldots \ldots \ldots \ldots, 42$

APPENDIX A. LITERATURE REVIEW $\ldots \ldots \ldots \ldots \ldots \ldots \ldots \ldots \ldots, 58$ 
APPENDIX B. DRAG CORRELATIONS .

B.0.1 Modified Ergun's equation and calculation of minimum fluidization velocity $\ldots \ldots \ldots \ldots \ldots \ldots \ldots \ldots, 66$

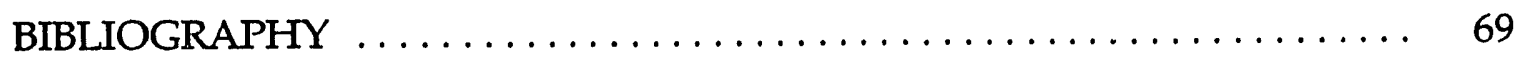




\section{LIST OF TABLES}

Table No.

Page

1 Particle and Fluid Characteristics $\ldots \ldots \ldots \ldots \ldots \ldots \ldots \ldots \ldots, 68$

2 Fluidized bed expansion characteristic $\ldots \ldots \ldots \ldots \ldots \ldots \ldots .68$ 


\subsection{INTRODUCTION}

\subsection{Fluidized Beds}

When a fluid flows upward through a bed of solid particles, the bed offers resistance. At low flow rates, the solid particles lie on one another and on the porous bottom of the column; they are said to be in a fixed state. As the velocity of flow increases, the drag force exerted on the particles increases. The particles rearrange themselves to offer less resistance to the fluid flow and the bed tends to expand. With subsequent increase in the upward fluid velocity, the expansion continues until the drag force exerted on the particles is sufficient to support their weight. The fluid/particle systems then begins to exhibit fluid-like properties and it flows under the influence of a hydrostatic pressure head. This is the point of incipient fluidization and the fluid velocity needed to achieve this is referred to as the minimum fluidization velocity. Just beyond this velocity, the pressure drop across the bed is approximately equal to the weight of the bed per unit area. At this point, the bed is more or less uniformly expanded and, up to this point, it makes little difference to the general bed behavior whether the fluid is a gas or a liquid.

A liquid fluidized bed continues to expand smoothly and stably with further increase in the flow velocity. This is termed particulately fluidized bed. However, of the fluid is a gas, the uniform expansion behavior is soon lost, as the gas velocity is increased, except with fine powders. Thus, for a gas-fluidized bed, instabilities develop and the bed is found to be in a state of continual and

\footnotetext{
*For a literature review on fluidization and the stability of uniformly fluidized bed, see appendix A.
} 
complex motion with rising regions of much reduced particle density called bubbles. This is termed aggregative fluidization. Over the flow range between incipient fluidization and the onset of bubbling, the bed is said to be in a quiescent state ( cf. Howard [ 1989 ] ). In either cases, as long as there is a clearly defined upper limit or surface to the bed, the bed is said to be in the state of dense-phase fluidization. With sufficient increase in the fluid flow rate this state soon converts into a fast or circulating state with entrainment of solids. The well defined upper surface of the bed disappears and the solid particles are carried out of the bed with the fluid stream.

The technique of fluidization is generally applicable to any particulate material in the size range between $10 \mu \mathrm{m}$ (diameter of the particle ) and $3 \mathrm{mrn}$, which includes the two classes, powders and granular solids. A powder is composed of particles up to $100 \mu \mathrm{m}$ with further subdivision into ultrafine ( 0.1 to $1.0 \mu \mathrm{m}$ ), superfine ( 1 to $10 \mu \mathrm{m}$ ), or granular ( 10 to $100 \mu \mathrm{m}$ ) particles. A granular solid consists of material ranging from about 100 to $3000 \mu \mathrm{m}$ (cf. Botterill [ 1975 ]).

One of the main practical advantages of fluidization is related to the "fluid-like" flowing properties of fluidized beds. Because of this unique feature, it is possible transport solid particles through pipes, to control the bed height by overflow or to circulate from one bed to another by gravity, avoiding the use of standard solid handling equipment.

In addition to their excellent transport properties, fluidized beds are an excellent medium for heat transfer. Intimate contact between the particles and the fluid is achieved because of the large particle surface area exposed to the fluid.

A fluidized bed can also be used to carry out chemical or physical reactions at uniform temperature ( $\mathrm{cf}$. Flood [ 1968 ]). For example, many metallic ores are roasted in fluidized beds for the removal of sulfur ( cf. Flood [ 1968 ]). Fluidized 
bed drying has found a wide use in the industry. In manufacturing certain chemicals, the usual operations of evaporation, crystallization, filtration, drying, and pulverization are replaced by a single process of drying in a fluidized bed (cf. Romankov [ 1971 ]).

These, and many other applications which apply the concepts of fluidization phenomenon, have motivated many researchers in the past to study this phenomenon. Of particular interest is finding the reasons for the differences in behavior between particulate and aggregative system of fluidization and in providing a criterion for distinguishing between these types of fluidization. This topic, and the hydrodynamic stability of fluid particle systems is discussed in the following section.

\subsection{Hydrodynamic Stability}

The essential problems of hydrodynamic stability were recognized and formulated in the nineteenth century, notably by Helmholtz, Kelvin, Hopf, Orr, Foa, Rayleigh and Reynolds. Since then, for nearly a century now, hydrodynamic stability has been recognized as one of the central areas of fluid mechanics. The question is when and how laminar flow breaks down into turbulent flow or some other laminar flow. Is the flow stable relative to infinitesimally small disturbances? (cf. Lin [ 1955 ]).

Suppose the system of hydrodynamic equation has a time independent solution

$$
u_{i}\left(x_{j}\right), \quad p\left(x_{j}\right), \quad \mathrm{T}\left(x_{j}\right),
$$

for the components of velocity, pressure and temperature. If a perturbed solution approaches the steady state solution(1-1) as time $\tau \rightarrow \infty$, the motion is termed 
asymptotically stable. There could be a case when the solution jumps to a different steady state and stays there. This situation is termed as metastable. However, our interest is to study the asymptotically stable situation. Hence, to solve the problem of hydrodynamic stability we assume that, for small disturbances the equations of motion may be linearized; that is, we shall neglect terms quadratic or higher in the disturbances and their derivatives. We also assume that the perturbation of each quantity can be resolved into independent components or modes varying with time $t$ like $e^{\text {st }}$ for some constant $s$, which is in general a complex quantity. The boundary conditions for the disturbances are usually homogeneous, resulting in a characteristic value problem with $s$ as a parameter. If all the characteristic values of $s$ have negative real parts, the motion is stable with respect to the infinitesimal disturbances. If some of the characteristic values have positive real parts, the motion is said to be unstable because a general initial small perturbation of the system would grow exponentially in time and when its amplitude attains a moderate size, the linearized equations are no longer applicable. Stokes, Kelvin and Rayleigh adapted this method of normal modes to fluid dynamics.

In order to understand the hydrodynamic stability of a system, it is necessary to understand the physical mechanism of instability. Broadly speaking, instability occurs because there is some disturbance of the equilibrium between the external forces, inertia, and viscous forces of a fluid. The buoyancy in a fluid of variable density, surface tension, magnetohydrodynamic forces, are some of the forces which govern the stability of the system. For example, if a heavy fluid is resting above a light fluid it is clear that the fluids tend to overturn under the action of gravity. A similar instability occurs on the free surface of a container of liquid when it is moved downwards at a uniform acceleration greater than the gravitational acceleration. If, in Couette flow, the circulation around the inner cylinder is greater in magnitude than that around the outer, the centrifugal force tends to throw out the fluid near the inner cylinder as an overturning 
instability.This centrifugal instability also may occur in flows along a rigid curved surface such as a concave wall of a channel. Surface tension resists the increase of area of a surface and generally exerts a stabilizing influence, particularly on disturbances of small length scale. A magnetic field can inhibit the motion of an electrically conducting fluid across the magnetic lines of force and thereby usually tends to stabilize flows. In a fluidized bed sources of instability can be due to the disturbance of the equilibrium between the drag force and the weight of the particle.

In the absence of any external force or of viscosity, a fluid moves according to the equilibrium between its inertia and pressure. A small disturbance may upset this equilibrium. This tendency of fluid to move down pressure gradients can amplify disturbances of certain flows and, thereby, create instability. An obvious effect of viscosity is to dissipate the energy of any disturbance, and hence, stabilize a flow. Indeed, for this reason any bounded flow is stable if the viscosity is large enough. Thermal conductivity, molecular diffusion of heat or a similar effect tends to smooth out the temperature differences of a disturbance and is usually a stabilizing influence. In general, acceleration of a laminar flow is stabilizing and deceleration is destabilizing. Finally, the boundaries of a flow are an important factor. They constrain the development of a disturbance and, usually, the closer they are together the more stable the flow. However, they sometimes give rise to strong shear in boundary layers which is diffused outwards by viscosity and so lead to instability of the flow. In a typical flow more than one of these mechanisms may act. This calls for a detailed analysis of a given problem. A linearized analysis will be able to explain some of this behavior. Although it is a necessary condition for stability, this is not sufficient. For a complete understanding a nonlinear analysis will be required.

The essential mathematical difference between fluid and particle dynamics is that the equations of motion are partial rather than ordinary differential 
equations. This difference leads to many technical difficulties in hydrodynamic stability, which, to this day, have been overcome for only a few classes of flows with very simple configuration. Many of the problems of practical interest are not simple to analyze. In principle, the motion of a system of particles suspended in a fluid is completely determined by the Navier-Stokes equations, if the fluid under question is linearly viscous. However, for a large number of closely spaced particles, as in a fluidized bed, the problem is far too complicated to permit direct solution when stated in these terms. Moreover, there are differences in opinion in modeling the quantities which appear in the equations of motion, e.g., stress tensors for the solid constituent, interaction between the constituents, etc. In the following section we will discuss these different points of view.

Stability analysis is a useful tool for any theory, enabling us to distinguish between solutions that are physically.realizable and others that are not. Great strides have been made in the study of stability of the motions of rigid bodies and deformable continuous media. In the latter sase, much, if not all, attention has been focused on the stability of a single constituent, whether it be a fluid ( $\mathrm{cf}$. Chandrasekhar [1981], Lin [1955], Orr [1907], Sommerfield [1908], Hopf [1957], Joseph [1976], Galdi and Padula [1990] ) or a solid (cf. Knops and Wilkes [1973] ). Very little effort has been expended in the study of the stability of flow of mixtures. This is not surprising in view of the fact that, until recently, there was not a mathematically rigorous theory for modeling the mechanics of mixtures ( cf. Truesdell [1984], [1957], Bowen [1976], Atkin and Craine [1976] ). Moreover, these theories lead to governing equations that are much more complicated than those for a single continuum. Of course, it is possible that for special flows these equations could reduce to a simple structure and provide some insight into the stability characteristica of the flow. One example, where some progress has been made in the study of the stability of flow of a mixture, is the stability of the state of uniform fluidization in the case of a fluid mixed with solid particles. 
If the volume fraction of the solid particles is large enough, then the mixture is sufficiently dense with the particles and both the constituents can be treated as continuum. The mixture is then regarded as the superposition of two "homogenized equivalent continua", in which one constituent can diffuse through the other ( cf. Truesdell [ 1957 ], [ 1984 ], Bowen [ 1976 ], Atkin and Craine [ 1976 ] ). Much of the recent work in multicomponent or multiphase mechanics is already in, or interpreted within the framework of such an approach.

Using mixture theory for modeling fluidization, El-Kaissy et. al. [ 1975 ] studied the stability of the state of uniform fiuidization. El-Kaissy et. al. [ 1976 ], Homsy et. al. [ 1980 ] modeled both the constituents as linearly viscous fluids. However, in the absence of the fluid, their model for the granular solid cannot predict the possibility of normal stress differences in simple shear flow, a phenomenon which is exhibited by granular materials.

The stability of the state of uniform fluidization has also been studied by Murray [ 1965 ], Pigford and Baron [ 1965 ], Jackson [ 1963 ], Anderson and Jackson [ 1968 ] Drew [ 1971 ] Grace and Tout [ 1979 ] etcetra. In order to make the problem amenable to analysis, Anderson and Jackson [ 1968 ] made several simplifying assumptions which reduceed the problem for determining the parameter which controls the linearized stability, to a quadratic equation. Anderson and Jackson [ 1968 ], like El-Kaissy, model the granular material as a linearly viscous fluid. This not withstanding, Anderson and Jackson [ 1968] are able to glean some very useful information regarding the stability of the state of uniform fluidization. They conjecture that the pressure-like term which appears in the constitutive equation for the solid could have an important role in determining the stability of the state of uniform fluidization, and we find, within the context of a more general stability study, that this is indeed true. The equations governing the linearized stability of the state of uniform fluidization 
can be written as a product of factors, one of which is precisely that which would be obtained were we to use an approximation similar to that of Anderson and Jackson [ 1968 ]. That is to say, if the state of uniform fluidization is unstable according to the criterion of Anderson and Jackson [ 1968 ], then it is also unstable according to the theory which we outline. Of course, it is possible that the system could be unstable to disturbances other than those delineated by the criterion of Anderson and Jackson [ 1968 ]. The studies of Anderson and Jackson [ 1968 ] has been followed by various experimental and theoretical studies of the stability of fludization, ( cf. Agarwal et. al. [ 1980 ], Batcheior [ 1988 ], Foscolo et. al. [ 1984 ], Foscolo et. al. [ 1987 ], Foscolo et. al [ 1985 ], Ganser et. al. [ 1990 ], Gibilaro et. al. [ 1986 ], Gidaspow et. al. [ 1983 ], Green et. al. [ 1987 a ], Green et. al. [ 1987 b ], Ham et. al. [ 1990 ], Homsy et. al. [ 1980 ], Liu [ 1983 ], Liu [ 1982 ], Massoudi [ 1988 ], Molerus [ 1967 ], Prosperetti et. al. [ 1987 ], Rietema et. al. [ 1990 ].

We assume that each constituent is dense enough in the mixture to be modeled as a "homogenized continuum". While the fluid constituent of the mixture is modeled as a linearly viscous fluid, an assumption consistent with all previous work, the solid constituent is modeled as a granular solid ( cf. Massoudi [ 1986 ]). Such an approach provides a specific constitutive structure for the pressure-like term of the solid constituent and obviates the necessity for ad hoc assumptions with regard to its relationship with the fluid pressure*, a necessity if we are to obtain a determinate system of equations in that there are as many equations as unknowns.

The balance laws for the mass and linear and angular momentum for each constituent have to be modified to account for the presence and the action of the other constituents. Thus, the theory requires constitutive equations for the

\footnotetext{
"This issue is discussed in detail elsewhere ( cf. Massoudi [ 1986 ], Rajagopal, Massoudi and Ekmann [ 1990 ]).
} 
interaction terms, which account for effects such as drag, virtual mass, lift, Basset forces, Magnus forces, Faxen forces etc. ( cf. Massoudi [ 1986 ], Johnson, Massoudi and Rajagopal [ 1990 ] ). Recently, using such an approach, several boundary value problems concerned with the flow of fluids mixed with solid particulates have been solved ( cf. Johnson, Massoudi and Rajagopal [ 1991 a ], [ 1991 b ], [ 1991 c ].

Using the mixture theory, Massoudi [ 1986 ] modeled the flow in a fluidized bed as the flow of a fluid mixed with a granular solid. He also showed that the equations governing the flow imbed most of the previous studies of the problem as special cases. The generality of his work is also its shortcoming in that, in incorporating all the effects that are relevant, Massoudi [ 1986 ] has so many a plethora of parameters characterizing the problem that gleaning information regarding the flow is very difficult.

In this paper we carry out a systematic linearized stability analysis of the state of uniform fluidization for a fluid mixed with granular particles. We carry out an optimization procedure which leads to bounds for certain parameters, within which the state of uniform fluidization is stable. We find that this stability depends critically on the structure of the pressure-like term as conjectured by Anderson and Jackson [ 1968 ], Jackson [ 1985 ].

We now outline the plan of this paper. In section 2, we introduce the notation and basic balance laws of mixture theory. In the next section, we introduce the constitutive relations for the stress in the granular material and the fluid, and also the interactive forces that come irito play in mixtures. Section 4 is devoted to deriving the basic governing equations and the appropriate nondimensionalization. This is followed, in section 5 , by the introduction of the state of uniform fluidization and the derivation of the equations governing the perturbations to the state of uniform fluidization. In section 6 , we carry out the 
usual normal node analysis. We simplify the analysis by considering disturbances only along the flow direction. In section 7, for the purpose of comparing our results with those of Anderson and Jackson [ 1968 ], we carry out a simplified stability analysis and show that the predictions of such a theory are completely in keeping with the predictions of the full theory. The final section is concerned with the full stability equations. 


\subsection{PRELIMINARIES AND BALANCE LAWS}

Before providing a brief review of the notation and the basic balance laws of the continuum theory of mixtures, we shall, for the sake of completeness, give a concise presentation of the developments in the area of fluidization.

Mathematical modeling of flows in fluidized beds use one of two approaches, averaging techniques ( cf. Anderson and Jackson [ 1967 ], [ 1968 ], [ 1963 ], [ 1985 ], Drew and Segel [ 1971 ], Murray [ 1965 ], [ 1967 ], Pigford and Baron [ 1965 ] ) or mixture theory ( cf. El-Kaissy [ 1975 ], [ 1976 ], Homsy et. al. [ 1980 ], Massoudi [ 1986 ], [ 1988 ] ). In either these approaches, the modeling of the fluid is essentially the same; differences are due to the constitutive equation used for the solid stress tensor and the interaction terms. A detailed discussion of the various models can be found in Massoudi and Boyle [ 1991 ]. A problem common to most of these approaches is the meaning and role of the term usually referred to as solid pressure ( cf. Prosperetti and Jones [ 1984 ], [ 1987 ], Drew and Segel [ 1971 ], Homsy et. al. [ 1980 ], Anderson and Jackson [ 1967 ], [ 1968 ], Givler [ 1987 ], Massoudi and Boyle [ 1991 ], Gidaspow [ 1983 ]). The derived equations are indeterminate in that there are more unknown than equations. The culprit is usually identified as the solid pressure and the difficulty is overcome by either setting the solid pressure equal to the fluid pressure or the difference between these pressures equal to a function of the volume fraction. Our approach is markedly different in that we have a specific constitutive relation for the spherical part of the solid stress ( an equation of state for the pressure like term ) which is appropriate for flowing granular materials. We thus model the flow in a fluidized bed as that due to mixture of a Newtonian fluid mixed with a granular solid. 
We now turn our attention to the balance laws of mixture theory ( $\mathrm{cf}$. Truesdell [ 1957 ], Bowen [ 1976 ], Truesdell [ 1984 ] ). We shall consider a mixture of a linearly viscous fluid $S_{f}$ with a granular solid $S_{s}$. At each instant of time, each point in space $x$ is occupied by particles belonging to both the constituents. Let $X_{s}$ and $X_{f}$ denote the positions of particles of $S_{s}$ and $S_{f}$ in their respective reference configurations. The motion of the solid $S_{s}$ and the fluid $S_{f}$ are defined through

$$
x_{s}=\chi_{s}\left(X_{s}, t\right), \quad x_{f}=\chi_{f}\left(X_{f}, t\right),
$$

These motions are assumed to be invertible and smooth enough that all the required derivatives are defined. The kinematical quantities associated with these motions are the velocities, velocity gradients and stretching tensors defined as

$$
\begin{aligned}
& \mathbf{v}_{s}=\frac{d_{s} \chi_{s}}{d t}, \quad \mathbf{v}_{f}=\frac{d_{f} \chi_{f}}{d t}, \\
& L_{s}=\operatorname{grad}_{s} v_{s}, \quad \cdot \quad L_{f}=\operatorname{grad}_{f} v_{f} \text {, }
\end{aligned}
$$

and,

$$
D_{s}=\frac{1}{2}\left[L_{s}+L_{s}^{T}\right], \quad D_{f}=\frac{1}{2}\left[L_{f}+L_{f}^{T}\right] .
$$

Here $\frac{d_{s}}{d t}$ and $\frac{d f}{d t}$ denote differentiation with respect to $t$, holding $X_{s}$ and $X_{\mathrm{f}}$ fixed, respectively, and a bold character designates a tensor (or vector ).

\subsection{Conservation of Mass}

We shall assume that there is no interconversion of mass between the two constituents and that the fluid and the solid are incompressible in the sense that their densities $\rho_{\mathrm{s} 0}$ and $\rho_{\mathrm{f} 0}$ are constant. Then, the bulk densities, $\rho_{\mathrm{s}}$ and $\rho_{\mathrm{f}}$, are given by 


$$
\begin{aligned}
& \rho_{s}(x, t)=\rho_{s 0} v, \\
& \rho_{f}(x, t)=\rho_{f 0}(1-v),
\end{aligned}
$$

where $v$ is the volume fraction of the solid constituent, $(0 \leq v<1)$. For a saturated mixture, $v=1-\varepsilon$, where $\varepsilon$ is the voidage. In the fluidized bed literature, these equations are usually expressed in terms of the voidage fraction, $\varepsilon$.

The appropriate form of the conservation of mass equation for the solid and fluid constituent are

$$
\begin{aligned}
& \frac{\partial \rho_{s}}{\partial t}+d i v_{s}\left(\rho_{s} \mathbf{v}_{s}\right)=0 \\
& \frac{\partial \rho_{f}}{\partial t}+d i v_{f}\left(\rho_{f} \mathbf{v}_{f}\right)=0
\end{aligned}
$$

respectively.

Substituting (2-5) and (2-6) into (2-7) and (2-8), yields simplified forms for conservation of mass, since $\rho_{s}$ and $\rho_{f}$ are constants.

$$
\begin{aligned}
& \frac{\partial v}{\partial t}+d i v_{s}\left(v \mathbf{v}_{s}\right)=0 \\
& \frac{\partial(1-v)}{\partial t}+d i v_{f}\left((1-v) \mathbf{v}_{\mathrm{f}}\right)=0
\end{aligned}
$$




\subsection{Conservation of Linear Momentum}

The balance of linear momentum for the two constituents can be written as

$$
\rho_{s} \frac{d \mathbf{v}_{s}}{d t}=\operatorname{div}\left(\mathbf{T}_{s}\right)-\mathbf{I}+\rho_{s} \mathbf{b}
$$

where

$$
\frac{d \mathbf{v}_{s}}{d t}=\frac{\partial \mathbf{v}_{\mathrm{s}}}{\partial t}+\left(\operatorname{grad}_{s}\left(\mathbf{v}_{s}\right)\right)\left(\mathbf{v}_{s}\right)
$$

and

$$
\rho_{f} \frac{d \mathbf{v}_{\mathbf{s}}}{d t}=d i v_{f}\left(\mathrm{~T}_{f}\right)+\mathbf{I}+\rho_{f} \mathbf{b}
$$

where

$$
\frac{d \mathbf{v}_{\mathbf{f}}}{d t}=\frac{\partial \mathbf{v}_{f}}{\partial t}+\left(\operatorname{grad}_{f} \mathbf{v}_{\mathrm{f}}\right) \mathbf{v}_{\mathbf{f}}
$$

$\mathbf{I}$ is the interaction between the solid and fluid constituent, $\mathbf{b}$ is the body force, $\mathbf{T}_{\mathbf{s}}$ and $T_{f}$ are the stress tensors for the solid and the fluid constituent respectively. We define the total stress, T, of the mixture as (cf. Green and Naghdi [ 1969 ])

$$
\mathbf{T}=\mathrm{T}_{s}+\mathrm{T}_{f}
$$

The balance of the moment of momentum for the mixture requires that the total stress for the mixture is symmetric ( assuming no body couples are present), i.e., $\mathbf{T}=\mathbf{T}^{\mathrm{T}}$. However, this is not necessarily true so for the partial stress tensors, $\mathbf{T}_{s}$ and $T_{f}$. 


\subsection{CONSTITUTIVE MODELING}

We model the fluidized bed as a mixture of granular material mixed with a linearly viscous fluid. ( $c$. Massoudi [1986]). We shall assume that the solid particles form a granular material whose Cauchy's stress tensor $\mathbf{T}_{\mathrm{s}}$ is given by (cf. Massoudi and Rajagopal [ 1990 ] ) Johnson et. al. [ 1991 ] ).

$$
\begin{aligned}
\mathrm{T}_{s}= & \mathrm{v}\left[\left(-p_{s}+\lambda_{s} \operatorname{tr}\left(\mathrm{D}_{s}\right)\right) \mathbf{1}+2 \mu_{s} \mathrm{D}_{s}\right] \\
& +\left[\beta_{1} \operatorname{grad}_{s} \mathrm{v} \cdot \operatorname{grad}_{s} \mathrm{v}\right] \mathbf{1}+\beta_{2} \operatorname{grad}_{s} \mathrm{v} \otimes \operatorname{grad}_{s} \mathrm{v},
\end{aligned}
$$

where, $\mathbf{1}$ is the identity tensor, $t r$ is the trace operator, $\mathrm{p}_{\mathrm{s}}$ plays a role akin to pressure in a compressible fluid, $\beta_{1}, \beta_{2}$ are material parameters that reflect the distribution of the granular solids, $\mu_{s}$ and $\lambda_{s}$ are granular viscosity coefficients (akin to the first and second coefficient of viscosity in a compressible fluid).

The constitutive equation for the partial stress tensor for the fluid constituent, $T_{f}$ is assumed to be (cf. Massoudi et. al. [ 1990 ]),

$$
\mathrm{T}_{f}=\left\{\left[-\hat{p}_{f}+\hat{\lambda}_{f} \operatorname{cr}\left(\mathrm{D}_{f}\right)\right] \mathbf{1}+2 \hat{\mu}_{f} \mathbf{D}_{f}\right\}
$$

where $\hat{p}_{f}$ is the fluid pressure, $\hat{\mu}_{f}$ and $\hat{\lambda}_{\mathrm{f}}$ are the first and second coefficient of viscosity of the fluid. While in general $\hat{\mathrm{p}}_{f}, \hat{\lambda}_{f}$, and $\hat{\mu}_{f}$ can be any functions of $v$, we shall assume them to be of the following form:

$$
\begin{aligned}
& \hat{p}_{f}=p_{f}(1-v), \\
& \hat{\lambda}_{f}=\lambda_{f}(1-v), \\
& \hat{\mu}_{f}=\mu_{f}(1-v),
\end{aligned}
$$


where $p_{f} \lambda_{f}$ and $\mu_{f}$ are assumed to be independent of $v$. With this we can rewrite equation (3-2) as

$$
\mathrm{T}_{f}=\left\{\left[-p_{f}+\lambda_{f} \operatorname{tr}\left(\mathbf{D}_{f}\right)\right] 1+2 \mu_{f} \mathbf{D}_{f}\right\}(1-v) .
$$

Since the total stress is defined as, $\mathbf{T}=\mathbf{T}_{s}+\mathbf{T}_{f}$, notice that when $\mathrm{v} \rightarrow 0$, $\mathbf{T} \rightarrow \mathbf{T}_{\mathbf{f}}$

We shall assume the constitutive equation for the interaction force $I$, is of the following form ( cf. Massoudi [ 1986 ], Drew and Segel [ 1971 ], Homsy et. al. [ 1980 ]),

$$
I=\alpha_{1} \operatorname{grad}_{s} \rho_{s}+\alpha_{2} \operatorname{grad}_{f} \rho_{f}+\alpha_{3}\left(\mathrm{v}_{s}-\mathrm{v}_{\mathrm{f}}\right)+\alpha_{4} \frac{D\left(\mathrm{v}_{\mathrm{s}}-\mathrm{v}_{\mathrm{f}}\right)}{D t}
$$

where,

$$
\begin{aligned}
& \frac{D\left(\mathrm{v}_{\mathrm{s}}-\mathrm{v}_{\mathrm{f}}\right)}{D t}=\left[\frac{d_{s}\left(\mathrm{v}_{\mathrm{s}}\right)}{d t}+\left(\operatorname{grad}_{s} \mathrm{v}_{\mathrm{s}}\right)^{T}\left(\mathrm{v}_{\mathrm{s}}-\mathrm{v}_{\mathrm{f}}\right)\right]-\left[\frac{d_{f}\left(\mathrm{v}_{\mathrm{f}}\right)}{d t}+\left(\operatorname{grad}_{f} \mathrm{v}_{\mathrm{f}}\right)^{T}\left(\mathrm{v}_{\mathrm{f}}-\mathrm{v}_{\mathrm{s}}\right)\right] \\
& \frac{d\left(\mathrm{v}_{s}\right)}{d t}=\frac{\partial\left(\mathrm{v}_{s}\right)}{\partial t}+\left(\operatorname{grad}_{s}\left(\mathrm{v}_{s}\right)\right)\left(\mathrm{v}_{s}\right),
\end{aligned}
$$

and

$$
\frac{d\left(\mathrm{v}_{f}\right)}{d t}=\frac{\partial\left(\mathrm{v}_{f}\right)}{\partial t}+\left(\operatorname{grad}_{f}\left(\mathrm{v}_{f}\right)\right)\left(\mathrm{v}_{f}\right)
$$

I incorporates buoyancy effects (the first and the second terms in (3-5)), the drag effect ( third term in (3-5)) and the virtual mass effect ( the last term in (3-5)). Other effects such as Faxen forces, Basset forces, Magnus effect can be easily incorporated and added to the system. The coefficients $\alpha_{1}, \alpha_{2}, \alpha_{3}$ and $\alpha_{4}$ must be determined experimentally and, in general, they depend on densities, viscosities, particle size, velocities, etc. Many forms of the relative acceleration term ( virtual 
mass effect ), which are not frame-indifferent, have been proposed. (cf. Drew et. al. [1979] and Johnson et. al. [1990] ). However, the form given in equation (3-6) is frame-indifferent.

The body force is taken to be

$$
\mathbf{b}=-g \mathbf{k}
$$

where $\mathbf{k}$ is the unit vector in the vertical direction. 


\subsection{BASIC GOVERNING EQUATIONS AND NON-DIMENSIONALIZATION}

For the sake of convenience ( to avoid too many subscripts), from now onwards, we would denote $v_{s}$ as $\mathbf{u}$ and $v_{f}$ as $\mathbf{v}$.

Let $d_{p}$ denote the average diameter of the particles and $V_{0}$ denote the characteristic velocity of the fluid. We introduce the following dimensionless quantities:

$$
\begin{aligned}
& \mathbf{x}^{*}=\frac{\mathbf{x}}{d_{p}}, \quad \mathbf{u}^{*}=\frac{\mathbf{u}}{V_{0}}, \quad \mathbf{v}^{*}=\frac{\mathbf{v}}{V_{0}}, \quad t^{*}=\frac{t V_{0}}{d_{p}}, \\
& p_{f}^{*}=\frac{p_{f}}{\rho_{f 0} V_{0}^{2}},
\end{aligned}
$$

We also define these non-dimensional material parameters.

$$
\begin{aligned}
& p_{s}^{0}=\frac{p_{s}}{\rho_{s 0} V_{0}^{2}}, \quad \alpha_{1}^{0}=\frac{\alpha_{1}}{V_{0}^{2}}, \quad \alpha_{2}^{0}=\frac{\alpha_{2}}{V_{0}^{2}}, \quad \alpha_{3}^{0}=\frac{\alpha_{3} d_{p}}{\rho_{s 0} V_{0}}, \quad \alpha_{4}^{0}=\frac{\alpha_{4}}{\rho_{s 0}}, \\
& \lambda_{s}^{0}=\frac{\lambda_{s}}{\rho_{s 0} V_{0} d_{p}}, \quad \lambda_{f}^{0}=\frac{\lambda_{f}}{\rho_{f 0} V_{0} d_{p}}, \quad \mu_{s}^{0}=\frac{\mu_{s}}{\rho_{s 0} V_{0} d_{p}}, \quad \mu_{f}^{0}=\frac{\mu_{f}}{\rho_{f 0} V_{0} d_{p}}, \\
& \beta_{1}^{0}=\frac{\beta_{1}}{\rho_{s 0} V_{0}^{2} d_{p}^{2}}, \quad \beta_{2}^{0}=\frac{\beta_{2}}{\rho_{s 0} V_{0}^{2} d_{p}^{2}}, \quad \rho_{f s}^{0}=\frac{\rho_{f 0}}{\rho_{s 0}}, \rho_{s f}^{0}=\frac{\rho_{s 0}}{\rho_{f 0}}, \quad g^{0}=\frac{g d_{p}}{V_{0}^{2}}
\end{aligned}
$$

We assume that the material parameters $\lambda_{s}{ }^{0}, \lambda_{f}{ }^{0}, \mu_{s}, \mu_{f}, \alpha_{1}{ }^{0}, \alpha_{2}{ }^{0}, \alpha_{3}{ }^{0}, \alpha_{4}{ }^{0}, \beta_{1}{ }^{0}$, $\beta_{2}{ }^{0}, \rho_{\mathrm{s} 0}, \rho_{\mathrm{f} 0}, \rho_{\mathrm{s}}{ }^{0}$ are constant. 


\subsection{Conservation of Mass for the Solid Constituent}

Inserting (4-1), into (2-9) yields

$$
\frac{\partial v}{\partial t^{*}}+\frac{\partial\left(v u_{j}^{*}\right)}{\partial x_{j}^{*}}=0
$$

\subsection{Conservation of Linear Momentum for the Solid Constituent}

Using (3-1), (3-5)-(3-7), (3-9), (4-1)-(4-5), we can rewrite (2-11) in the nondimensional form

$$
\begin{aligned}
& v\left[\frac{\partial u_{i}^{*}}{\partial t^{*}}+u_{j}^{*} \frac{\partial u_{i}^{*}}{\partial x_{j}^{*}}\right]=-p_{s}^{0} \frac{\partial v}{\partial x_{i}^{*}}+\beta_{1}^{0} \frac{\partial}{\partial x_{i}^{*}}\left(\frac{\partial v}{\partial x_{m}^{*}} \frac{\partial v}{\partial x_{m}^{*}}\right)+\lambda_{s}^{0} \frac{\partial}{\partial x_{i}^{*}}\left(v \frac{\partial u_{m}^{*}}{\partial x_{m}^{*}}\right) \\
& +\beta_{2}^{0} \frac{\partial}{\partial x_{j}^{*}}\left(\frac{\partial v}{\partial x_{i}^{*}} \frac{\partial v}{\partial x_{j}^{*}}\right)+\mu_{s}^{0} \frac{\partial}{\partial x_{j}^{*}} v\left\{\frac{\partial u_{i}^{*}}{\partial x_{j}^{*}}+\frac{\partial u_{j}^{*}}{\partial x_{i}^{*}}\right\}-\alpha_{1}^{0} \frac{\partial v}{\partial x_{i}^{*}}+\alpha_{2}^{0} \rho_{f s}^{0} \frac{\partial v}{\partial x_{i}^{*}} \\
& -\alpha_{3}^{0}\left(u_{i}^{*}-v_{i}^{*}\right)-\alpha_{4}^{0}\left[\frac{\partial\left(u_{i}^{*}-v_{i}^{*}\right)}{\partial t^{*}}+u_{j}^{*} \frac{\partial u_{i}^{*}}{\partial x_{j}^{*}}-v_{j}^{*} \frac{\partial v_{i}^{*}}{\partial x_{j}^{*}}\right. \\
& \left.+\left(u_{j}^{*}-v_{j}^{*}\right) \frac{\partial\left(u_{j}^{*}+v_{j}^{*}\right)}{\partial x_{i}^{*}}\right]-g^{0} v \delta_{i 3} .
\end{aligned}
$$

The dimensionless numbers which appear in the equations represent various competing physical effects namely, viscosity, inertia, gravity, volume distribution, cohesion and adhesion. These numbers provide a means for studying the effect of the various parameters in a systematic manner. By neglecting appropriate dimensionless numbers and focusing on just one or two of them, we can obtain some idea about how these non-dimensional numbers (and the physical quantities that they represent) affect the flow. 


\subsection{Conservation of Mass for the Fluid Constituent}

Substituting (4-1) into (2-10) we find that

$$
\frac{\partial(1-v)}{\partial t^{*}}+\frac{\partial\left((1-v) v_{j}^{*}\right)}{\partial x_{j}^{*}}=0
$$

\subsection{Conservation of Linear Momentum for the Fluid Constituent}

Using equations (3-2), (3-5)-(3-7), (3-9), and (4-1)-(4-5) we can rewrite (2-12) in the non-dimensional form as

$$
\begin{aligned}
& (1-v)\left[\frac{\partial v_{i}^{*}}{\partial t^{*}}+v_{j}^{*} \frac{\partial v_{i}^{*}}{\partial x_{j}^{*}}\right]=-\frac{\partial}{\partial x_{i}^{*}}\left(p_{f}^{*}(1-v)\right)+\lambda_{f}^{0}\left[\frac{\partial}{\partial x_{i}^{*}}\left\{(1-v) \frac{\partial v_{m}^{*}}{\partial x_{m}^{*}}\right\}\right] \\
& +\mu_{f}^{0}\left[\frac{\partial}{\partial x_{j}^{*}}\left\{(1-v)\left(\frac{\partial v_{i}^{*}}{\partial x_{j}^{*}}+\frac{\partial v_{j}^{*}}{\partial x_{i}^{*}}\right)\right\}\right]+\alpha_{1}^{0} \frac{1}{\rho_{f s}^{0}} \frac{\partial v}{\partial x_{i}^{*}}-\alpha_{2}^{0} \frac{\partial v}{\partial x_{i}^{*}} \\
& +\alpha_{3}^{0}\left(u_{i}^{*}-v_{i}^{*}\right)+\alpha_{4}^{0}\left[\frac{\partial\left(u_{i}^{*}-v_{i}^{*}\right)}{\partial t^{*}}+u_{j}^{*} \frac{\partial u_{i}^{*}}{\partial x_{j}^{*}}\right. \\
& \left.-v_{j}^{*} \frac{\partial v_{i}^{*}}{\partial x_{j}^{*}}+\left(u_{j}^{*}-v_{j}^{*}\right) \frac{\partial\left(u_{j}^{*}+v_{j}^{*}\right)}{\partial x_{i}^{*}}\right]-g^{0}(1-v) \delta_{i 3} .
\end{aligned}
$$

Equations (4-6), (4-7), (4-8) and (4-9) provides a system of 8 equations for 8 unknowns, the velocities of the two constituents $u, v$, the fluid pressure $p_{f}$ and the volume distribution function $v$. 


\subsection{STATE OF UNIFORM FLUIDIZATION}

The simplest solution of the governing equations is one corresponding to a steady state of uniform fluidization where the local velocity of the solid particles is zero, and the local velocity of the Auid is constant and directed vertically upward. The volume fraction is assumed to be constant. Thus,

$$
\mathbf{u}=\mathbf{0}, \mathbf{v}=\mathbf{v}_{0}=V_{0} \mathbf{k}, \mathbf{v}=\mathrm{v}_{0} \text {, }
$$

where $\mathbf{k}$ is the unit vector in the vertical direction and $\mathrm{V}_{0}$ and $\mathrm{v}_{0}$ are constants. Thus, for the basic flow

$$
\mathbf{u}^{*}=0, \quad \mathbf{v}^{*}=1 \mathbf{k} \quad .
$$

For the purpose of convenience and clarity we shall drop the superscript $*$ and denote $\mathbf{x}^{*}, \mathbf{u}^{*}$ etc. as $\mathbf{x}, \mathbf{u}$ etc. respectively. 


\subsection{PERTURBANCES TO THE STATE OF UNIFORM FLUIDIZATION}

We shall now consider the stability of the state of uniform fluidization subject to small perturbations. We shall assume that

$$
\begin{aligned}
& \mathbf{u}=\varepsilon \mathbf{u}_{1}, \mathbf{v}=1 \mathbf{k}+\varepsilon \mathbf{v}_{1}, \\
& p_{f}=p_{0}+\varepsilon p_{1} \text { and } v=v_{0}+\varepsilon v_{1},
\end{aligned}
$$

where the quantities with subscript 0 correspond to the basic flow while quantities with subscript 1 correspond to the perturbation. Since we are interested in small perturbations, we shall assume that $\varepsilon \ll 1$.

Substituting equations (6-1) and (6-2) into equations (4-6), (4-7), (4-8) and (4-9), neglecting quantities of order $O\left(\varepsilon^{2}\right)$, and equating the terms of the same order, we obtain the governing equations of order 0 and 1 .

\subsection{Equations of Order $\varepsilon^{0}$}

The equations governing the mass conservation of the solid and fluid constituents are automatically satisfied at the zeroth order. The $x$ and $y$ momentum equations for the fluid constituent results in

$$
\frac{\partial p_{0}}{\partial x}=0 \text { and } \frac{\partial p_{0}}{\partial y}=0
$$

The only non-trivial components of the momentum equations are the equation in the $z$-direction; for the solid constituent it is 


$$
\alpha_{3}^{0}-g^{0} \cdot v_{0}=0^{*}
$$

and for the fluid constituent is

$$
\alpha_{3}^{0}+\rho_{f s}^{0}\left[\frac{\partial p_{0}}{\partial z}+g^{0}\right]\left(1-v_{0}\right)=0
$$

\subsection{Equations of Order $\varepsilon^{1}$}

To first order, the conservation of mass for the solid constituent is ( in the indicial notation )

$$
\frac{\partial v_{1}}{\partial t}+v_{0} \frac{\partial u_{1_{i}}}{\partial x_{i}}=0
$$

while that for the fluid is ( in the indicial notation )

$$
\frac{\partial v_{1}}{\partial t}-\left(1-v_{0}\right) \frac{\partial v_{1_{i}}}{\partial x_{i}}+\frac{\partial v_{1}}{\partial z}=0
$$

The balance of linear momentum for solid constituent is

$$
\begin{aligned}
& v_{0} \frac{\partial u_{1_{i}}}{\partial t}=\alpha_{4}{ }^{0}\left[\frac{\partial u_{1_{z}}}{\partial x_{i}}+\frac{\partial v_{1_{z}}}{\partial x_{i}}+\frac{\partial v_{1_{i}}}{\partial z}\right]-\alpha_{4}{ }^{0}\left[\frac{\partial\left(\mathrm{u}_{1_{i}}-\mathrm{v}_{1_{i}}\right)}{\partial t}\right] \\
& -\alpha_{3}^{0}\left[u_{1_{i}}-v_{1_{i}}\right]+\left[\mu_{s}^{0}+\lambda_{s}^{0}\right] v_{0} \frac{\partial}{\partial x_{i}}\left(\frac{\partial u_{1_{j}}}{\partial x_{j}}\right)+\mu_{s}^{0} v_{0}\left[\frac{\partial^{2}}{\partial x_{j} \partial x_{j}} u_{1_{i}}\right]
\end{aligned}
$$

"This immediately provides an expression for the coeff. of drag as $\alpha_{3}=\frac{g v_{0} \rho_{s}}{v_{0}}$. For related comments, see Appendix A. 
24

$$
+\left[-p_{s}^{0}+\alpha_{2}^{0} \rho_{f s}^{0}-\alpha_{1}^{0}\right] \frac{\partial v_{1}}{\partial x_{i}}-g^{0} v_{1} \delta_{i 3}
$$

The balance of linear momentum for the fluid constituent is

$$
\begin{aligned}
& \left(v_{0}-1\right) \frac{\partial v_{1_{i}}}{\partial t}=\rho_{s f}^{0} \alpha_{4}^{0}\left[\frac{\partial u_{1_{z}}}{\partial x_{i}}+\frac{\partial v_{1_{z}}}{\partial x_{i}}+\frac{\partial v_{1_{i}}}{\partial z}\right]-\alpha_{4}^{0} \rho_{s f}^{0}\left[\frac{\partial\left(u_{1_{i}}-v_{1_{i}}\right)}{\partial t}\right] \\
& -\alpha_{3}^{0} \rho_{s f}^{0}\left[u_{1_{i}}-v_{1_{i}}\right]-\left[\mu_{f}^{0}+\lambda_{f}^{0}\right]\left(1-v_{0}\right) \frac{\partial}{\partial x_{i}}\left(\frac{\partial v_{1_{j}}}{\partial x_{j}}\right) \\
& -\mu_{f}^{0}\left(1-v_{0}\right)\left[\frac{\partial^{2} v_{1_{i}}}{\partial x_{j} \partial x_{j}}\right]-\left[p_{0}-\alpha_{2}^{0}+\frac{1}{\rho_{f s}^{0}} \alpha_{1}^{0}\right] \frac{\partial v_{1}}{\partial x_{i}}+\left(1-v_{0}\right) \frac{\partial p_{1}}{\partial x_{i}} \\
& +\left(1-v_{0}\right) \frac{\partial v_{1_{i}}}{\partial z}-\left[g^{0}+\frac{\partial p_{0}}{\partial z}\right] v_{1} \delta_{i 3}
\end{aligned}
$$




\subsection{STABILITY ANALYSIS}

If $\phi$ is a perturbation, then we shall assume that ( cf. Lin [ 1955])

$$
\phi(\mathbf{x}, t)=\phi_{p} e^{s t} e^{i \sigma \cdot x},
$$

where $\phi$ is unknown, $\phi_{\mathrm{p}}$ is the amplitude of the disturbance, $x$ is the position vector, $\mathrm{i}=\sqrt{-1}, \sigma$ is a vector whose components are wave numbers (real) and $s=\zeta+i \omega$.

Here $\omega$ is related to the velocity of wave propagation $\mathrm{V}$ through

$$
V=-\frac{\omega}{|\sigma|}
$$

The negative sign implies that a positive value for $\mathrm{V}$ corresponds to waves propagating in the positive $x$ direction. The factor $\zeta$ determines whether the waves are amplified or damped. If $\zeta<0$, the disturbance will die out and the flow will be stable to the linearized disturbances; if $\zeta=0$, the flow is neutrally stable; and if $\zeta>0$, the disturbance will grow and the flow is unstable.

From equation (7-1), We can write the perturbation quantities as

$$
\begin{aligned}
& \mathbf{u}_{1}=\mathbf{u}_{p} e^{s t} e^{i \sigma \cdot \mathbf{x}}, \quad \mathbf{v}_{1}=\mathbf{v}_{p} e^{s t} e^{i \sigma \cdot \mathbf{x}}, \\
& p_{1}=p_{p} e^{s t} e^{i \sigma \cdot \mathbf{x}}, \quad v_{1}=v_{p} e^{s t} e^{i \sigma \cdot x} .
\end{aligned}
$$

Combining equation (6-3) and (6-4) and using the expressions for $\rho_{\mathrm{fs}}{ }^{0}, \alpha_{3}{ }^{0}$, and $\mathrm{g}^{0}$, we obtain the following equation (in the dimensional terms): 


$$
g \rho_{s 0} v_{0}+g \rho_{f 0}\left(1-v_{0}\right)=-\frac{\partial p_{0}}{\partial z}\left(1-v_{0}\right)
$$

Note that equation (6-3) asserts that the drag force on the particles is balanced by the weight of the particles and does not seem to take the buoyancy force into account. This is not so and should be apparent from the following explanation.

The fundamental assumption of mixture theory is that at any instant of time every point in the space is occupied by each constituent. Hence when we are using the continuum theory, we cannot invoke the concept of a particle, suspended in a fluid, on which various forces act. In the continuum theory, we derive that the drag force due to the flow of the fluid is balanced by just the weight of the particles, but appropriate homogenizations for the two constituents takes care of the fact that one constituent displaces the other and this is reflected in the homogenized values for the densities. However, if we take into account the buoyancy forces in (7-5) once again, the errors are quite significant.

Substituting equations (7-3) and (7-4) into (6-5) - (6-8) and simplifying, we obtain a set of eight homogeneous linear algebraic equations in the eight unknowns, $\mathbf{u}_{\mathrm{p}}$,

$$
\begin{aligned}
& \mathbf{v}_{\mathrm{p}}, \mathrm{p}_{\mathrm{p}} \text {, and } \mathrm{v}_{\mathrm{p}} \text {, } \\
& i v_{0}\left[\sigma_{x} u_{p_{x}}+\sigma_{y} u_{p_{y}}+\sigma_{z} u_{p_{z}}\right]+s v_{p}=0 \\
& i\left(v_{0}-1\right)\left[\sigma_{x} v_{p_{x}}+\sigma_{y} v_{p_{y}}+\sigma_{z} v_{p_{z}}\right]+\left(s+i \sigma_{z}\right) v_{p}=0 \\
& {\left[-\alpha_{3}^{0}-\alpha_{4}^{0} s-v_{0} s-\mu_{s}^{0} v_{0}\left(\sigma_{x}^{2}+\sigma_{y}^{2}+\sigma_{z}^{2}\right)-v_{0} \sigma_{x}{ }^{2}\left(\mu_{s}^{0}+\lambda_{s}^{0}\right)\right] u_{p_{x}}} \\
& -\left[\left(\mu_{s}^{0}+\lambda_{s}^{0}\right) v_{0} \sigma_{x} \sigma_{y}\right] u_{p_{y}}+\left[i \alpha_{4}^{0} \sigma_{x}-\left(\mu_{s}^{0}+\lambda_{s}^{0}\right) v_{0} \sigma_{x} \sigma_{z}\right] u_{p_{z}} \\
& +\left[\alpha_{3}{ }^{0}+\alpha_{4}{ }^{0}\left(s+i \sigma_{z}\right)\right] \mathrm{v}_{p_{x}}+\left[i\left(\alpha_{2}{ }^{0} \rho_{f s}^{0}-p_{s}^{0}-\alpha_{1}{ }^{0}\right) \sigma_{x}\right] v_{p} \\
& +\left[i \alpha_{4}{ }^{0} \sigma_{x}\right] \mathrm{v}_{p_{z}}=0
\end{aligned}
$$




$$
\begin{aligned}
& -\left[\left(\mu_{s}^{0}+\lambda_{s}^{0}\right) v_{0} \sigma_{x} \sigma_{y}\right] u_{p_{x}} \\
& +\left[-\alpha_{3}{ }^{0}-\alpha_{4}{ }^{0} s-v_{0} s-\mu_{s}^{0} v_{0}\left(\sigma_{x}{ }^{2}+\sigma_{y}{ }^{2}+\sigma_{z}{ }^{2}\right)-v_{0} \sigma_{y}{ }^{2}\left(\mu_{s}{ }^{0}+\lambda_{s}{ }^{0}\right)\right] u_{p_{y}} \\
& +\left[i \alpha_{4}^{0} \sigma_{y}-\left(\mu_{s}^{0}+\lambda_{s}^{0}\right) v_{0} \sigma_{y} \sigma_{z}\right] u_{p_{z}}+\left[\alpha_{3}^{0}+\alpha_{4}{ }^{0}\left(s+i \sigma_{z}\right)\right] v_{p_{y}} \\
& +\left[i\left(\alpha_{2}^{0} \rho_{f s}^{0}-p_{s}^{0}-\alpha_{1}^{0}\right) \sigma_{y}\right] v_{p}+\left[i \alpha_{4}^{0} \sigma_{y}\right] v_{p_{z}}=0 \\
& -\left[\left(\mu_{s}^{0}+\lambda_{s}^{0}\right) v_{0} \sigma_{x} \sigma_{z}\right] u_{p_{x}}-\left[\left(\mu_{s}^{0}+\lambda_{s}^{0}\right) v_{0} \sigma_{y} \sigma_{z}\right] u_{p_{y}} \\
& +\left[-\alpha_{3}{ }^{0}-\alpha_{4}{ }^{0} s-v_{0} s-\mu_{s}{ }^{0} v_{0}\left(\sigma_{x}{ }^{2}+\sigma_{y}{ }^{2}+\sigma_{z}{ }^{2}\right)\right. \\
& \left.-v_{0} \sigma_{z}{ }^{2}\left(\mu_{s}^{0}+\lambda_{s}{ }^{0}\right)+i \alpha_{4}^{0} \sigma_{z}\right] u_{p_{z}}+\left[\alpha_{3}{ }^{0}+\alpha_{4}{ }^{0}\left(s+2 i \sigma_{z}\right)\right] v_{p_{z}} \\
& +\left[i\left(\alpha_{2}^{0} \rho_{f s}^{0}-p_{s}^{0}-\alpha_{1}^{0}\right) \sigma_{z}-g^{0}\right] v_{p}=0 \\
& {\left[-\alpha_{4}^{0} \rho_{s f}^{0} s-\alpha_{3}^{0} \rho_{s f}^{0}\right] u_{p_{x}}+\left[i \alpha_{4}^{0} \rho_{s f}^{0} \sigma_{x}\right] u_{p_{z}}} \\
& +\left[\alpha_{3}^{0} \rho_{s f}^{0}+\alpha_{4}^{0} \rho_{s f}^{0} s-\left(v_{0}-1\right)\left(s+i \sigma_{z}+\left(\mu_{f}^{0}+\lambda_{f}^{0}\right) \sigma_{x}^{2}\right.\right. \\
& \left.\left.+\mu_{f}^{0}\left(\sigma_{x}^{2}+\sigma_{y}^{2}+\sigma_{z}^{2}\right)\right)+i \alpha_{4}^{0} \rho_{s f}^{0} \sigma_{z}\right] v_{p_{x}} \\
& +\left[-\left(\mu_{f}^{0}+\lambda_{f}^{0}\right)\left(v_{0}-1\right) \sigma_{x} \sigma_{y}\right] v_{p_{y}}+\left[-i \sigma_{x}\left(p_{0}+\alpha_{1}^{0} \rho_{s f}^{0}-\alpha_{2}^{0}\right)\right] v_{p} \\
& -\left[\left(v_{0}-1\right) i \sigma_{x}\right] p_{p}+\left[-\left(\mu_{f}^{0}+\lambda_{f}^{0}\right)\left(v_{0}-1\right) \sigma_{x} \sigma_{z}+i \alpha_{4}^{0} \rho_{s f}^{0} \sigma_{x}\right] v_{p_{z}}=0 \\
& {\left[-\alpha_{4}{ }^{0} \rho_{s f}{ }^{0} s-\alpha_{3}{ }^{0} \rho_{s f}^{0}\right] u_{p_{y}}+\left[i \alpha_{4}^{0} \rho_{s f}^{0} \sigma_{y}\right] u_{p_{z}}} \\
& -\left[\left(\mu_{f}^{0}+\lambda_{f}^{0}\right)\left(v_{0}-1\right) \sigma_{x} \sigma_{y}\right] \mathrm{v}_{p_{x}}+\left[\alpha_{3}^{0} \rho_{s f}^{0}+\alpha_{4}{ }^{0} \rho_{s f}{ }^{0} s-\left(v_{0}-1\right)\left(s+i \sigma_{z}\right.\right. \\
& \left.\left.+\left(\mu_{f}^{0}+\lambda_{f}^{0}\right) \sigma_{y}^{2}+\mu_{f}^{0}\left(\sigma_{x}^{2}+\sigma_{y}^{2}+\sigma_{z}^{2}\right)\right)+i \alpha_{4}^{0} \rho_{s f}^{0} \sigma_{z}\right] v_{p_{y}} \\
& +\left[-i \sigma_{y}\left(p_{0}+\alpha_{1}^{0} \rho_{s f}^{0}-\alpha_{2}^{0}\right)\right] v_{p}-\left[\left(v_{0}-1\right) i \sigma_{y}\right] p_{p} \\
& +\left[-\left(\mu_{f}^{0}+\lambda_{f}^{0}\right)\left(v_{0}-1\right) \sigma_{y} \sigma_{z}+i \alpha_{4}{ }^{0} \rho_{s f}^{0} \sigma_{y}\right] v_{p_{z}}=0
\end{aligned}
$$




$$
\begin{aligned}
& {\left[-\alpha_{4}{ }^{0} \rho_{s f}^{0} s-\alpha_{3}{ }^{0} \rho_{s f}^{0}\right] u_{p_{z}}+\left[i \alpha_{4}{ }^{0} \rho_{s f}{ }^{0} \sigma_{z}\right] u_{p_{z}}} \\
& -\left[\left(\mu_{f}^{0}+\lambda_{f}^{0}\right)\left(v_{0}-1\right) \sigma_{x} \sigma_{z}\right] \mathrm{v}_{p_{x}}+\left[-\left(\mu_{f}^{0}+\lambda_{f}^{0}\right)\left(v_{0}-1\right) \sigma_{y} \sigma_{z}\right] \mathrm{v}_{p_{y}} \\
& +\left[\alpha_{3}^{0} \rho_{s f}^{0}+\alpha_{4}{ }^{0} \rho_{s f}^{0} s-\left(v_{0}-1\right)\left(s+i \sigma_{z}+\left(\mu_{f}^{0}+\lambda_{f}^{0}\right) \sigma_{z}{ }^{2}\right.\right. \\
& \left.\left.+\mu_{f}^{0}\left(\sigma_{x}{ }^{2}+\sigma_{y}{ }^{2}+\sigma_{z}{ }^{2}\right)\right)+2 i \alpha_{4}{ }^{0} \rho_{s f}^{0} \sigma_{z}\right] \mathrm{v}_{p_{z}} \\
& +\left[-i\left(v_{0}-1\right) \sigma_{z}\right] p_{p}+\left[-g^{0}-\frac{\partial p_{0}}{\partial z}-i \sigma_{z}\left(p_{0}+\alpha_{1}^{0} \rho_{s f}^{0}-\alpha_{2}^{0}\right)\right] v_{p}=0
\end{aligned}
$$

where $u_{p}$ is the amplitude vector for the perturbation of the velocity of the solid constituent, $\mathbf{v}_{\mathrm{p}}$ is the amplitude vector for the perturbation of the velocity of the fluid constituent, $\mathrm{p}_{\mathrm{p}}$ is the amplitude vector of the perturbed fluid pressure, and $v_{p}$ is the amplitude vector of the perturbed volume distribution function.

Now, consider disturbances which are propagating only in the z-direction, i.e., the direction in which the fluid is flowing. In this case, the $x$ and $y$ components of the wave number, $\sigma_{x}$ and $\sigma_{y}$ are zero. Then, the set of equations (7-6) - (7-13) reduce to the following set of eight simple homogeneous algebraic equations:

$$
\begin{aligned}
& A_{1} u_{p_{z}}+s v_{p}=0 \\
& A_{2} \mathrm{v}_{p_{z}}+\left(s+A_{3}\right) \mathrm{v}_{p}=0 \\
& \left(A_{4}+A_{5} s\right) u_{p_{x}}+\left(A_{6}+A_{7} s\right) \mathrm{v}_{p_{x}}=0 \\
& \left(A_{4}+A_{5} s\right) u_{p_{y}}+\left(A_{6}+A_{7} s\right) \mathrm{v}_{p_{y}}=0 \\
& \left(A_{8}+A_{5} s\right) u_{p_{z}}+\left(A_{9}+A_{7} s\right) \mathrm{v}_{p_{z}}+A_{10} v_{p}=0 \\
& \left(A_{11}+A_{12} s\right) u_{p_{x}}+\left(A_{13}+A_{14} s\right) \mathrm{v}_{p_{x}}=0 \\
& \left(A_{11}+A_{12} s\right) u_{p_{y}}+\left(A_{13}+A_{14} s\right) \mathrm{v}_{p_{y}}=0 \\
& \left(A_{15}+A_{12} s\right) u_{p_{z}}+\left(A_{16}+A_{14} s\right) \mathrm{v}_{p_{z}}+A_{17} v_{p}+A_{2} p_{p}=0 .
\end{aligned}
$$

where the $A^{\prime}$ s in equations (7-14) are given by, 


$$
\begin{aligned}
& A_{1}=i v_{0} \sigma_{z} \text {, } \\
& A_{2}=-i\left(1-v_{0}\right) \sigma_{z}, \\
& A_{3}=i \sigma_{z} \text {, } \\
& A_{4}=-\alpha_{3}{ }^{0}-\mu_{s}{ }^{0} v_{0} \sigma_{z}{ }^{2} \\
& A_{5}=-v_{0}-\alpha_{4}{ }^{0} \text {, } \\
& A_{6}=\alpha_{3}{ }^{0}+i \alpha_{4}{ }^{0} \sigma_{2} \text {, } \\
& A_{7}=\alpha_{4}^{0} \text {, } \\
& A_{8}=-\alpha_{3}{ }^{0}-v_{0} \sigma_{z}{ }^{2}\left(2 \mu_{s}{ }^{0}+\lambda_{s}{ }^{0}\right)+i \alpha_{4}{ }^{0} \sigma_{z} \text {, } \\
& A_{9}=2 i \alpha_{4}{ }^{0} \sigma_{2}+\alpha_{3}{ }^{0} \text {, } \\
& A_{10}=-g^{0}+i\left(-p_{s}^{0}-\alpha_{1}^{0}+\alpha_{2}^{0} \rho_{f s}^{0}\right) \sigma_{2}, \\
& A_{11}=-\alpha_{3}^{0} \rho_{s f}^{0} \text {, } \\
& A_{12}=-\alpha_{4}^{0} \rho_{s f}^{0} \text {, } \\
& A_{13}=\alpha_{3}^{0} \rho_{s f}^{0}-\left(v_{0}-1\right) i \sigma_{z}+i \alpha_{4}^{0} \rho_{s f}^{0} \sigma_{z}-\mu_{f}^{0} \sigma_{z}^{2}\left(v_{0}-1\right) \text {, } \\
& A_{14}=-\left(v_{0}-1\right)+\alpha_{4}^{0} \rho_{s f}^{0} \text {, } \\
& A_{15}=-\alpha_{3}^{0} \rho_{s f}^{0}+i \sigma_{z} \alpha_{4}^{0} \rho_{s f}^{0} \text {, } \\
& A_{16}=\alpha_{3}^{0} \rho_{s f}^{0}-i\left(v_{0}-1\right) \sigma_{z}+2 i \alpha_{4}{ }^{0} \rho_{s f}^{0} \sigma_{z}-\left(v_{0}-1\right)\left(\lambda_{f}^{0}+2 \mu_{f}^{0}\right) \sigma_{z}{ }^{2} \text {, } \\
& A_{17}=i\left(\alpha_{2}^{0}-\alpha_{1}^{0} \rho_{s f}^{0}-p_{0}\right) \sigma_{z}-\frac{g^{0} v_{0}}{\left(1-v_{0}\right) \rho_{f s}^{0}} \text {. }
\end{aligned}
$$

Thus with these assumptions the equations, governing the stability of a uniformly fluidized bed reduce to a problem of the form

$$
\mathbf{N}\left(\sigma_{z}, s\right) \mathbf{W}=\mathbf{0},
$$

where, 


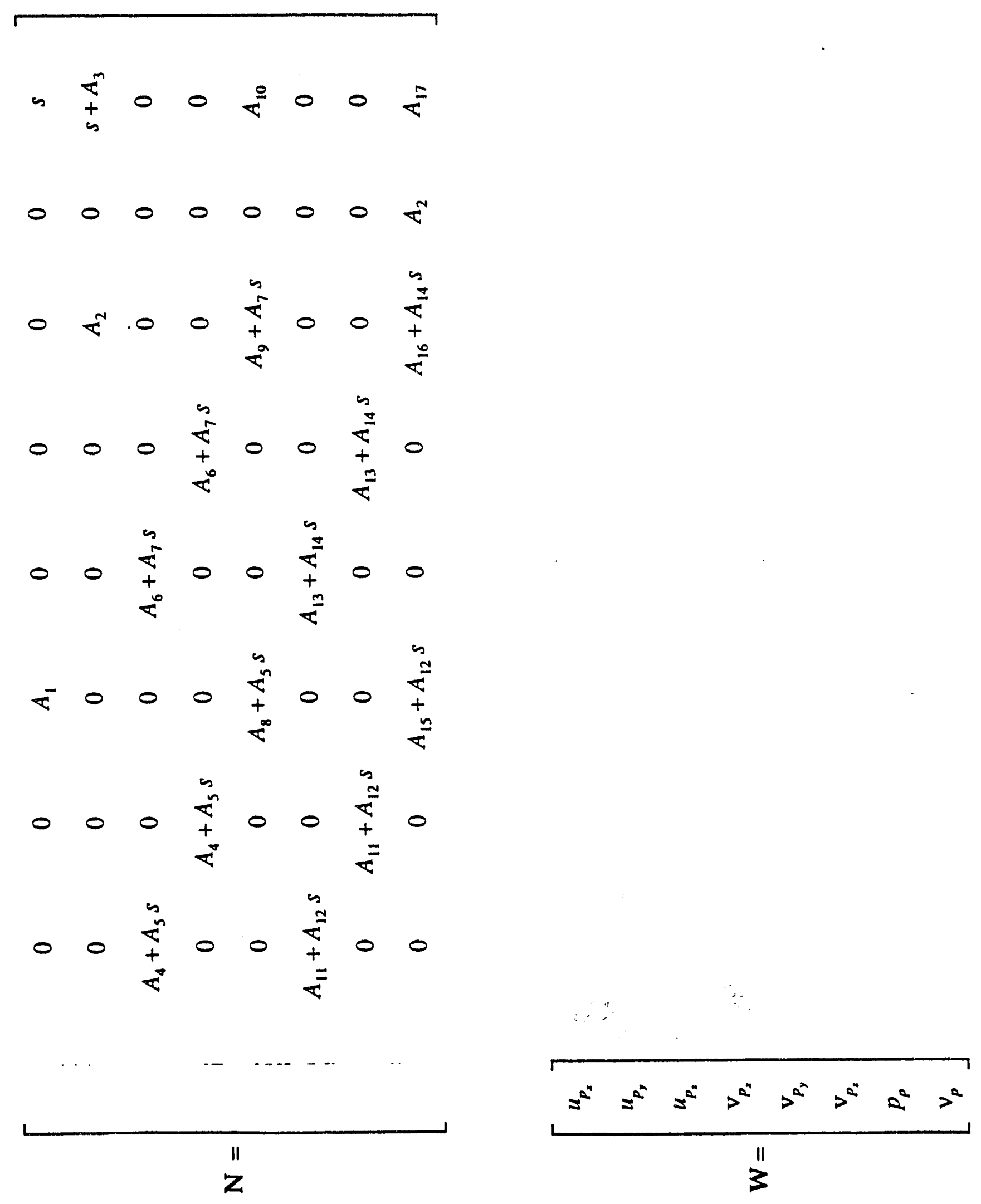


where $\mathbf{N}$ is an eight by eight matrix and $\mathbf{W}$ is a vector in eight unknown quantities*

Equation (7-16) implies that in order to have a non-trivial solution of $W$, the determinant of coefficient matrix, i.e., $\operatorname{det}(\mathbf{N})$, should be zero. This would give a characteristic polynomial equation in powers of $s$. The roots of the polynomial will determine the stability of our problem.

As $\mathbf{N}$ is an $8 \times 8$ matrix, a regular expansion to calculate the determinant is a tedious process. However, the determinant of a matrix is not changed by interchanging the rows and by adding a multiple of one row to another. Thus, using Gaussian elimination, we transform the matrix $\mathbf{N}$ into an upper triangular matrix, for which the determinant is the product of the diagonal elements. Thus, we obtain the following characteristic equation:

$$
\begin{aligned}
& {\left[A_{10}-\frac{s}{A_{1}}\left(A_{8}+A_{5} s\right)-\frac{\left(s+A_{3}\right)}{A_{2}}\left(A_{9}+A_{7} s\right)\right]} \\
& {\left[\left[A_{4}+A_{5} s\right]\left[A_{13}+A_{14} s\right]-\left(A_{6}+A_{7} s\right)\left(A_{11}+A_{12} s\right)\right]^{2}=0,}
\end{aligned}
$$

which yields,

$$
\begin{aligned}
& {\left[\left(-A_{2} A_{5}-A_{1} A_{7}\right) s^{2}-\left(A_{2} A_{8}+A_{1} A_{9}+A_{1} A_{3} A_{7}\right) s\right.} \\
& \left.+\left(A_{1} A_{2} A_{10}-A_{1} A_{3} A_{9}\right)\right] \\
& {\left[\left(A_{14} A_{5}-A_{7} A_{12}\right) s^{2}+\left(A_{4} A_{14}+A_{13} A_{5}-A_{6} A_{12}-A_{7} A_{11}\right) s\right.} \\
& \left.+\left(A_{13} A_{4}-A_{6} A_{11}\right)\right]^{2}=0 .
\end{aligned}
$$

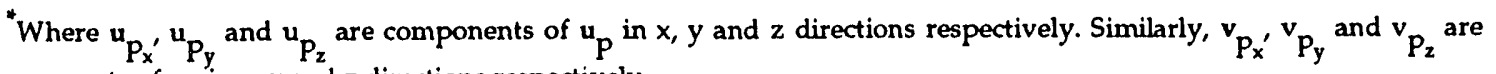
components of $v_{p}$ in $x, y$ and $z$ directions respectively.
} 
Equation (7-18) is the characteristic equation in $s$. This is a product of two equations, which are quadratic in s. Hence, equation (7-18) can be rewritten as

$$
\begin{aligned}
& {\left[\left(-A_{2} A_{5}-A_{1} A_{7}\right) s^{2}-\left(A_{2} A_{8}+A_{1} A_{9}+A_{1} A_{3} A_{7}\right) s\right.} \\
& \left.+\left(A_{1} A_{2} A_{10}-A_{1} A_{3} A_{9}\right)\right]=0,
\end{aligned}
$$

or

$$
\begin{aligned}
& {\left[\left(A_{14} A_{5}-A_{7} A_{12}\right) s^{2}+\left(A_{4} A_{14}+A_{13} A_{5}-A_{6} A_{12}-A_{7} A_{11}\right) s\right.} \\
& \left.+\left(A_{13} A_{4}-A_{6} A_{11}\right)\right]=0
\end{aligned}
$$

A careful investigation of these equations reveals that, for the same structure for all the appropriate parameters as considered by Anderson and Jackson [ 1968 ], Equation (7-19), reduces to an equation similar in structure to the characteristic equation obtained by them*. Thus, if the flow is unstable within the context of the equation similar to that obtained by Anderson and Jackson, the flow is unstable within the context of the full theory as these are sufficient conditions for instability. An analysis similar to that of Anderson and Jackson [ 1968 ] will provide sufficient conditions for the instability of the whole system. We shall see later that the stability of the system is completely determined by (7-19) ( the Anderson and Jackson like equation ), the other equation not predicting any instability of the perturbation. We shall discuss this issue in detail in the next section.

"The details are in the next section. 


\subsection{A SIMPLIFIED STABILITY ANALYSIS}

Before we carry out the linear stability analysis, we shall carry out a simplified linearized stability similar to that of Anderson and Jackson, which reduces the characteristic equation governing the linearized stability analysis to a quadratic equation similar in structure to (7-19). Ofcourse, our equations are somewhat different from the previous work in that the structure for our models are different. However, we find that the results are qualitatively in keeping $u$ ith the analysis of Anderson and Jackson, as is to be expected, as our general equations imbed the governing equations of Anderson and Jackson. Our general characteristic equation is an eight order equation. Interestingly, we find that if the interaction term has a special.form and if the wave vector has only the component in the direction of the flow, we can reduce the characteristic equation to a fifth order equation by using Gaussian elimination.

Now in order to compare our equations and theory with the earlier work of Anderson and Jackson, we use a procedure similar to theirs to obtain a quadratic equation in the growth number. We set parameters $\alpha_{1}{ }^{0}, \alpha_{2}{ }^{0}$ and $\alpha_{4}{ }^{0}$ to zero. These dimensionless numbers are due to the structure of our model for the interaction term. In order to compare our results with the earlier works, we have to drop the dimensionless number associated with the virtual mass term, $\alpha_{4}{ }^{0}$. Dimensionless numbers, $\alpha_{1}{ }^{0}$ and $\alpha_{2}{ }^{0}$ appear due to the buoyancy effects, which we again set to zero to consider the same form of interaction as considered by previous researchers. We apply the divergence operator to both sides of equation (6-7) to obtain the following equation: 


$$
\begin{aligned}
& -v_{0} \frac{\partial}{\partial t}\left(\frac{\partial u_{1_{i}}}{\partial x_{i}}\right)-p_{s}^{0} \frac{\partial}{\partial x_{i}}\left(\frac{\partial v_{1}}{\partial x_{i}}\right)+\left(\lambda_{s}^{0}+2 \mu_{s}^{0}\right) v_{0} \frac{\partial}{\partial x_{i}}\left(\frac{\partial}{\partial x_{i}}\right)\left(\frac{\partial u_{1_{m}}}{\partial x_{m}}\right) \\
& -\alpha_{3}^{0}\left(\frac{\partial u_{1_{i}}}{\partial x_{i}}-\frac{\partial v_{1_{i}}}{\partial x_{i}}\right)-g^{0} \frac{\partial v_{1}}{\partial z}=0 .
\end{aligned}
$$

From the conservation of mass equations (6-5) and (6-6) we have,

$$
\frac{\partial v_{1}}{\partial t}=-v_{0} \frac{\partial u_{1}}{\partial x_{j}}, \quad \frac{\partial v_{1}}{\partial t}+\frac{\partial v_{1}}{\partial z}=\left(1-v_{0}\right) \frac{\partial v_{1_{j}}}{\partial x_{j}}
$$

Substituting equation (8-2) into (8-1) we obtain

$$
\begin{aligned}
& \frac{\partial}{\partial t}\left(\frac{\partial v_{1}}{\partial t}\right)-p_{s}^{0} \frac{\partial}{\partial x_{i}}\left(\frac{\partial v_{1}}{\partial x_{i}}\right)-\left(\lambda_{s}^{0}+2 \mu_{s}^{0}\right) \frac{\partial}{\partial x_{i}}\left(\frac{\partial}{\partial x_{i}}\right)\left(\frac{\partial v_{1}}{\partial t}\right) \\
& -\alpha_{3}{ }^{0}\left[-\frac{1}{v_{0}} \frac{\partial v_{1}}{\partial t}-\frac{1}{\left(1-v_{0}\right)} \frac{\partial v_{1}}{\partial t}-\frac{1}{\left(1-v_{0}\right)} \frac{\partial v_{1}}{\partial z}\right]-g^{0} \frac{\partial v_{1}}{\partial z}=0 .
\end{aligned}
$$

Now equation (8-3) is a partial differential equation in $v_{1}$. As before, in order to solve the above equation, we assume the disturbances to be spatially periodic. Thus we assume the following form for $v_{1}$ :

$$
v_{1}=v_{p} e^{s t} e^{i \sigma_{2} x_{3}}
$$

where $v_{p}$ is the amplitude of disturbance for the volume fraction, $s$ is the growth constant and $\sigma_{z}$ is the wave number in the $z$ direction. Then, it follows from equation (8-4) that

$$
\begin{aligned}
& \frac{\partial v_{1}}{\partial t}=v_{1} s \quad, \frac{\partial^{2} v_{1}}{\partial t^{2}}=v_{1} s^{2}, \quad \frac{\partial v_{1}}{\partial z}=i v_{1} \sigma_{z}, \\
& \frac{\partial}{\partial z}\left(\frac{\partial v_{1}}{\partial z}\right)=\frac{\partial^{2} v_{1}}{\partial z^{2}}=-v_{1} \sigma_{2}{ }^{2},
\end{aligned}
$$

and hence, 


$$
\begin{aligned}
& v_{1}\left[s^{2}+\left\{\sigma_{2}^{2}\left(\lambda_{s}^{0}+2 \mu_{s}^{0}\right)+\frac{\alpha_{3}^{0}}{v_{0}\left(1-v_{0}\right)}\right\} s\right. \\
& \left.+\left\{\frac{\alpha_{3}^{0}}{\left(1-v_{0}\right)}-g^{0}-i p_{s}^{0} \sigma_{3}\right\} i \sigma_{z}\right]=0,
\end{aligned}
$$

which is the form we obtain from the equation (7-19) by substituting the appropriate form of the nondimensional parameters, $A^{\prime} s$, from equation (7-15) within the context of our specific assumptions ( for the values of the material parameters ). This equation resembles the equation of Anderson and Jackson [ 1968 ] in the sense that it has the same structure as obtained by them. 


\subsection{STABILIZING ROLE OF THE SPHERICAL PART OF THE STRESS (PRESSURE-LIKE TERM)}

In order to carry out a systematic study of the stability of the flow, we need to have a good idea of the values of the various non-dimensional numbers which appear in the governing equations. While some of these are concerned with the structure of the granular materials, others can be obtained by knowing the fluid properties and by correlating with experimental results which are already available. There are various ways in which we can obtain the material moduli appearing in the partial stress tensor for the granular material. We have a crude idea about the sign and the order of most of these material functions, but there is no precise experimental information. The sign and the order of the parameters related to the viscosity and the second coefficient of viscosity can usually be obtained from thermodynamical considerations. However, we have no such information about an important parameter, which appears in the equation of state for the pressure like term. In our analysis, the material function $\mathrm{p}_{s}^{0}$ plays the role of pressure. It has been conjectured that this function could play a stabilizing role. We obtain bounds for the range of this important parameter which stabilizes the state of uniform fluidization with respect to infinitesimal perturbances. This can be done by optimizing the root of the characteristic equation for stability with respect to the wave number.

We observe ( numerically) that for the range of parameters considered", the roots of the quadratic equation (7-20) always have negative real parts. Thus, we are primarily concerned with the predictions of (7-19), which, interestingly enough,

"Range of the parameters can be found from the figures $1-13$ 
is the counterpart of the equation of Anderson and Jackson. The roots of equation (7-19) sometimes have negative real part (stable), and sometimes positive real part (unstable), depending upon the range of parameters considered.

We rewrite equation (7-19) in the dimensional parameters as follows:

$$
s^{2}+(C+D i) s+(E+F i)=0,
$$

where,

$$
\begin{aligned}
& C=\left[\frac{\alpha_{3}{ }^{0}+v_{0}\left(1-v_{0}\right)\left(2 \mu_{s}{ }^{0}+\lambda_{s}{ }^{0}\right) \sigma_{z}{ }^{2}}{\alpha_{4}{ }^{0}+v_{0}\left(1-v_{0}\right)}\right] \\
& D=\left[\frac{-\alpha_{4}{ }^{0}\left(1-4 v_{0}\right) \sigma_{z}}{\alpha_{4}{ }^{0}+v_{0}\left(1-v_{0}\right)}\right] \\
& E=\left[\frac{-\left[2 \alpha_{4}{ }^{0}+\left(\alpha_{2}{ }^{0} \rho_{f s}{ }^{0}-p_{s}{ }^{0}-\alpha_{1}{ }^{0}\right)\left(1-v_{0}\right)\right] v_{0} \sigma_{z}{ }^{2}}{\alpha_{4}{ }^{0}+v_{0}\left(1-v_{0}\right)}\right] \\
& F=\left[\frac{\left[\alpha_{3}{ }^{0}-g^{0}\left(1-v_{0}\right)\right] v_{0} \sigma_{3}}{\alpha_{4}{ }^{0}+v_{0}\left(1-v_{0}\right)}\right]
\end{aligned}
$$

The roots of equation (9-1) are given by:

$$
s=\frac{-(C+D i) \pm \sqrt{(C+D i)^{2}-4(E+F i)}}{2}
$$

and are given by

$$
\begin{aligned}
& s_{1}=\frac{(-C+X)+i(Y-D)}{2} \\
& s_{2}=\frac{(-C-X)-i(Y+D)}{2}
\end{aligned}
$$

where $X$ and $Y$ are defined by

$$
\sqrt{(C+D i)^{2}-4(E+F i)}=X+i Y .
$$


Due to the values of $C, D, E$ and $F$, it is possible that either $X$ or $Y$, or both are zero. We have to examine the individual cases. When both of them are zero, then the real part of the root of the characteristic equation is always negative. Hence, we have stability. The other two cases are listed below.

If $Y \neq 0$, that is if the left hand side of equation (9-5) is complex, then $X$ can be expressed as:

$$
X=\frac{C D-2 F}{Y},
$$

and hence $Y$ is given by

$$
Y=\sqrt{\frac{-\left(C^{2}-D^{2}-4 E\right)+\sqrt{\left(C^{2}-D^{2}-4 E\right)^{2}+4(C D-2 F)^{2}}}{2}} .
$$

However, if $Y=0$, then $X$ is given by,

$$
X=\sqrt{\left(C^{2}-D^{2}-4 E\right)} .
$$

From the above equation (9-4) it is clear that when $X>C$, we will obtain a positive real root for the characteristic $s$ which implies instability of the system. 
Interestingly in both the cases (9-6) and (9-7)*, after a lengthy calculation, we find that the condition for $X>C$ reduces to,

$$
E C^{2}<F^{2}-C D F
$$

or,

$$
h_{4}<\frac{h_{5}{ }^{2} h_{6}-h_{5} h_{3}\left(h_{2}+h_{1} \sigma_{z}{ }^{2}\right)}{\left(h_{1} \sigma_{z}{ }^{2}+h_{2}\right)^{2}}
$$

where,

Equation (9-8) is also applicable for the case when $Y=0$. At first glance it appears that the condition $X>C$ reduces to $D^{2}+4 E<0$. However, when $Y=0$, We have, $C D=2 \mathrm{~F}$ and using this relation, we can show that the condition, $D^{2}+4 E<0$, is the same as given by equation (9-8). Because, by substituting for $D$ as,

$$
D=\frac{2 F}{C},
$$

in,

$$
D^{2}+4 E<0 \text {. }
$$

we have, since, $C D=2 F$

$$
F^{2}+E C^{2}<0
$$

or

$$
E C^{2}<-F^{2}
$$

or

$$
E C^{2}<F^{2}-(2 F) F,
$$
Since $C D=2 F$, we obtain,

$$
E C^{2}<F^{2}-C D F,
$$
which is equation (9-8). 


$$
\begin{aligned}
& h_{1}=v_{0}\left(1-v_{0}\right)\left(2 \mu_{s}^{0}+\lambda_{s}^{0}\right), \\
& h_{2}=\alpha_{3}^{0}, \\
& h_{3}=-\alpha_{4}^{0}\left(1-4 v_{0}\right), \\
& h_{4}=-\left[2 \alpha_{4}^{0}+\left(\alpha_{2}^{0} \rho_{f s}^{0}-p_{s}^{0}-\alpha_{1}^{0}\right)\left(1-v_{0}\right)\right] v_{0}, \\
& h_{5}=\left[\alpha_{3}^{0}-g^{0}\left(1-v_{0}\right)\right] v_{0} \\
& h_{6}=\alpha_{4}^{0}+v_{0}\left(1-v_{0}\right)
\end{aligned}
$$

Equation (9-9) gives the condition which determines the sign and range for the nondimensional parameters $\mathrm{p}_{\mathrm{s}}^{0}$ etc. If all but the parameter $\mathrm{p}_{\mathrm{s}}^{0}$ are assigned values, then this reduces to a condition on $\mathrm{p}_{\mathrm{s}}^{0}$ which is concerned with the pressure like term in the equation. But this equation involves a parameter $\sigma_{z^{\prime}}$ which is not a material parameter, that has to be determined separately. In order to obtain a bound for this parameter, we find the extremum value of this parameter such that the stability condition is met. We obtain $\sigma_{z}$ by differentiating the root of the characteristic equation (9-1) and equating it to zero, i.e.,

$$
\text { Real part of }\left(\frac{d s}{d \sigma_{z}}\right)=0 \text {, }
$$

where,

$$
2 s=-(C+D i) \pm \sqrt{(C+D i)^{2}-4(E+F i)}
$$

This procedure results in the following equation for $\sigma_{z}{ }^{2}$ :

$$
\left(\sigma_{z}^{2}\right)_{\text {extreme }}=\frac{\left(h_{2} h_{3} h_{5}-h_{5}{ }^{2} h_{6}\right)}{\left(h_{1} h_{3} h_{5}-h_{3}{ }^{2} h_{4}-4 h_{4}{ }^{2} h_{6}+4 h_{1} h_{2} h_{4}\right)}
$$

Substituting equation (9-11) into equation (9-9), we obtain:

$$
m_{5} h_{4}^{5}+m_{4} h_{4}^{4}+m_{3} h_{4}^{3}+m_{2} h_{4}^{2}+m_{1} h_{4}+m_{0}>0
$$

where, 


$$
\begin{aligned}
m_{5}= & -16 h_{2}{ }^{2} h_{6}{ }^{2} \\
m_{4}= & 16 h_{5}{ }^{2} h_{6}{ }^{3}-16 h_{2} h_{3} h_{5} h_{6}{ }^{2}-8 h_{2}{ }^{2} h_{3}{ }^{2} h_{6}+32 h_{1} h_{2}{ }^{3} h_{6}, \\
m_{3}= & 8 h_{3}{ }^{2} h_{5}{ }^{2} h_{6}{ }^{2}-40 h_{1} h_{2} h_{5}{ }^{2} h_{6}{ }^{2}-8 h_{2} h_{3}{ }^{3} h_{5} h_{6} \\
& +48 h_{1} h_{2}{ }^{2} h_{3} h_{5} h_{6}-h_{2}{ }^{2} h_{3}{ }^{4}+8 h_{1} h_{2}{ }^{3} h_{3}{ }^{2}-16 h_{1}{ }^{2} h_{2}{ }^{4} \\
m_{2}= & -12 h_{1} h_{3} h_{5}{ }^{3} h_{6}{ }^{2}+2 h_{1} h_{2} h_{3}{ }^{2} h_{5}{ }^{2} h_{6}+h_{3}{ }^{4} h_{5}{ }^{2} h_{6}-h_{2} h_{3}{ }^{5} h_{5} \\
& +24 h_{1}{ }^{2} h_{2}{ }^{2} h_{5}{ }^{2} h_{6}+12 h_{1} h_{2}{ }^{2} h_{3}{ }^{3} h_{5}-32 h_{1}{ }^{2} h_{2}{ }^{3} h_{3} h_{5} \\
m_{1}= & -h_{1}{ }^{2} h_{5}{ }^{4} h_{6}{ }^{2}-3 h_{1} h_{3}{ }^{3} h_{5}{ }^{3} h_{6}+16 h_{1}{ }^{2} h_{2} h_{3} h_{5}{ }^{3} h_{6} \\
& -16 h_{1}{ }^{2} h_{2}{ }^{2} h_{3}{ }^{2} h_{5}{ }^{2}+3 h_{1} h_{2} h_{3}{ }^{4} h_{5}{ }^{2} \\
m_{0}= & 2 h_{1}{ }^{2} h_{3}{ }^{2} h_{5}{ }^{4} h_{6}-2 h_{1}{ }^{2} h_{2} h_{3}{ }^{3} h_{5}{ }^{3}
\end{aligned}
$$

Equation (9-12) is the condition which ensures positive real roots for the characteristic equation which, in turn, implies that our system is unstable. We will have to solve the equation (9-12) numerically to obtain bounds on the material function $p_{s}^{0}$, i.e., the values of $\left(p_{s}{ }^{0}\right)_{\max }$ and $\left(p_{s}^{0}\right)_{\min }$. 


\subsection{RESULTS AND CONCLUSION}

Our primary interest in this paper is to discuss the effect of the pressure-like term on the linearized stability of the state of uniform fluidization. The term $\mathrm{p}_{\mathrm{s}}{ }^{0}$ is the non-dimensional number which is the ratio of the pressure effects to the inertial effects. We consider the following parameters* ( cf. Ham [ 1990 ]) for our parametric study: $\rho_{\mathrm{fo}}=1.00 \mathrm{~g} / \mathrm{cm}^{3}, \rho_{\mathrm{s} 0}=2.42 \mathrm{~g} / \mathrm{cm}^{3}, \mathrm{~d}_{\mathrm{p}}=0.066 \mathrm{~cm}$, $v_{0}=0.59, \mu_{f}=0.01$ poise, $\alpha_{1}{ }^{0}=0.50, \alpha_{2}{ }^{0}=0.50, \alpha_{4}{ }^{0}=0.50$, $\mu_{s}=10.0$ poise. Using these values for the material parameter we use the optimization procedure to obtain the maximum and minimum values for the parameter $\mathrm{p}_{\mathrm{s}}^{0}$ within which the state of uniform fluidization is stable. Figure 1, shows how the real part of $s$ varies with the wave number $\sigma_{\mathrm{z}}$. We find that the values of $\mathrm{p}_{\mathrm{s}}{ }^{0}$ inside the bound, the real part is negative and the flow is stable for all values of the wave numbers. However, for values of $\mathrm{p}_{\mathrm{s}}{ }^{0}$ outside the bound, namely $\mathrm{p}_{\mathrm{s}}{ }^{0}=-10.0,-5.0,0.0$ the flow is unstable as the real part is positive. Figure 2, depicts the behavior for values of $\mathrm{p}_{\mathrm{s}}{ }^{0}$ within the bounds ( extreme values of $\mathrm{p}_{\mathrm{s}}{ }^{0}$ ), for larger absolute values of $\mathrm{p}_{\mathrm{s}}{ }^{0}$, for which the flow is stable for all values of the wave numbers. In figure 3 , we see that for value of $\mathrm{p}_{\mathrm{s}}{ }^{0}>\left(\mathrm{p}_{\mathrm{s}}\right)_{\text {max }}$ the flow is not stable to all wave numbers and becomes unstable after an initial value of the wave number $\sigma_{\mathrm{cr}}$. Yet another example, where the flow is stable for values of wave numbers below a critical value but unstable for values above the critical wave number, is provided by figure 4 . We find that by increasing the value of the non-dimensional number $\mathrm{p}_{\mathrm{s}}{ }^{0}$ even further results in the flow being unstable to all perturbations ( $c$ f. figure 5 ). Thus, we see quite clearly that the parameter $\mathrm{p}_{\mathrm{s}}{ }^{0}$, and thus in turn, $\mathrm{p}_{\mathrm{s}}$, have a profound effect on the nature of the stability of the state of uniform fluidization.

"These parameters are for the case when the liquid in question is water and the particles are glass beads 
Figure 6 portrays the stability curves for the case ( the particles have higher density i.e., they are heavier than the previous considered ) $\rho_{\mathrm{s} 0}=2.49 \mathrm{~g} / \mathrm{cm}^{3}$, $\mathrm{d}_{\mathrm{p}}=0.066 \mathrm{~cm}, \mathrm{v}_{0}=0.59, \mu_{\mathrm{f}}=0.01$ poise $, \alpha_{1}{ }^{0}=0.5, \alpha_{2}{ }^{0}=0.5, \alpha_{4}{ }^{0}=0.5$, $\mu_{s}=10.0$ poise and $p_{s}{ }^{0}=1000.0$. Notice that the value of the non-dimensional number $p_{s}{ }^{0}$ is within our bounds and we expect the flow to be stable. We notice however that increasing the density of the fluid has a destabilizing effect in that the stability curves move closer to the $\sigma$-axis. Figure 7 depicts the stability curves for varying fluid density, when $\mathrm{p}_{\mathrm{s}}{ }^{0}$ is outside the bounds. Figure 8 portrays the stability curves for the case $\rho_{\mathrm{fo}}=1.08 \mathrm{~g} / \mathrm{cm}^{3}, \mathrm{~d}_{\mathrm{p}}=0.066 \mathrm{~cm}, \mathrm{v}_{0}=0.59$, $\mu_{f}=0.01$ poise, $\alpha_{1}{ }^{0}=0.5, \alpha_{2}{ }^{0}=0.5, \alpha_{4}{ }^{0}=0.5, \mu_{s}=10.0$ poise and $\mathrm{p}_{\mathrm{s}}{ }^{0}=1000.0$, we see how the stability curves change with increasing solid density. We find that by increasing the solid density, all other material constants being held constant, leads to greater stability in that the stability curves move away from the $\sigma$-axis.

The volume fraction of the solid is expected to have a significant effect on the state of uniform fluidization. Intuitively, we would expect that increasing the volume fraction of the solid should lead to a more stable situation. Figure 9 verifies that this is indeed the case when we are in the unstable regime in the sense that $p_{s}{ }^{0}$ lies outside the bounds. When $p_{s}{ }^{0}$ is inside the bounds, figure 10 verifies that the effect is just the opposite, that is increasing the volume fraction is destabilizing.

When $\mathrm{p}_{\mathrm{s}}{ }^{0}$ is within our bounds, we find that increasing the particle diameter has a stabilizing influence on the flow up to a certain diameter, in the special case depicted in figure 11.

The first counter intuitive results are obtained by varying the fluid viscosity but holding other material parameters fixed. We see that while the flow is stable, increasing the viscosity makes it less stable in that the stability curves shift 
towards the $\sigma$-axis ( cf. figure 12 ). This is however not to be totally unexpected, for even in the theory of stability of a single component compressible fluid such a result obtains ( cf. Galdi and Padula [ 1990 ] ). Similar behavior is noticed when the solid viscoisty term is varied keeping other material parameters fixed ( $\mathrm{cf}$. figure 13 ).

\section{ACKNOWLEDGEMENT:}

K. R. Rajagopal thanks the Department of Energy for supporting this work through a grant through the Morgantown Energy Technology Center. 


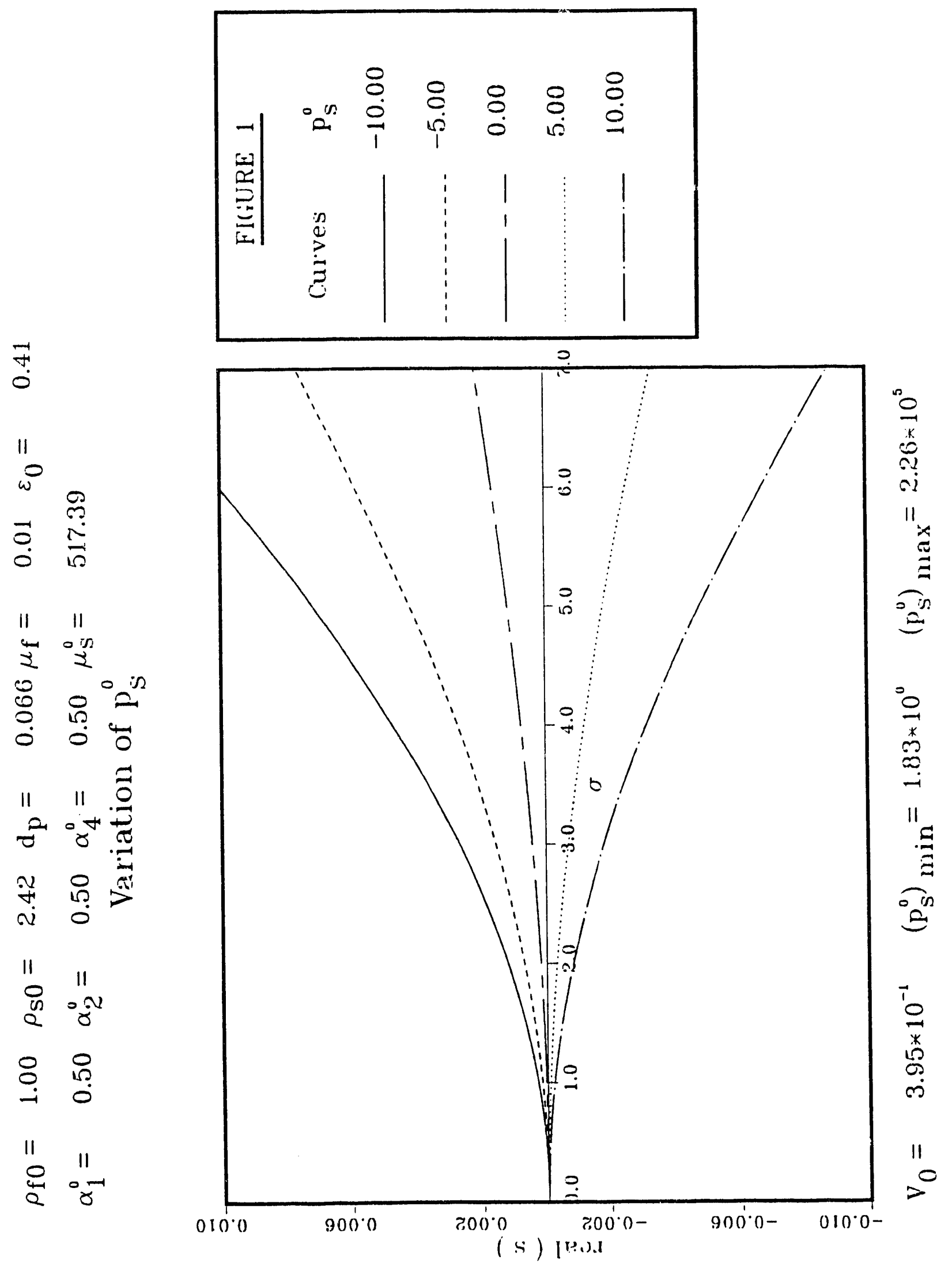




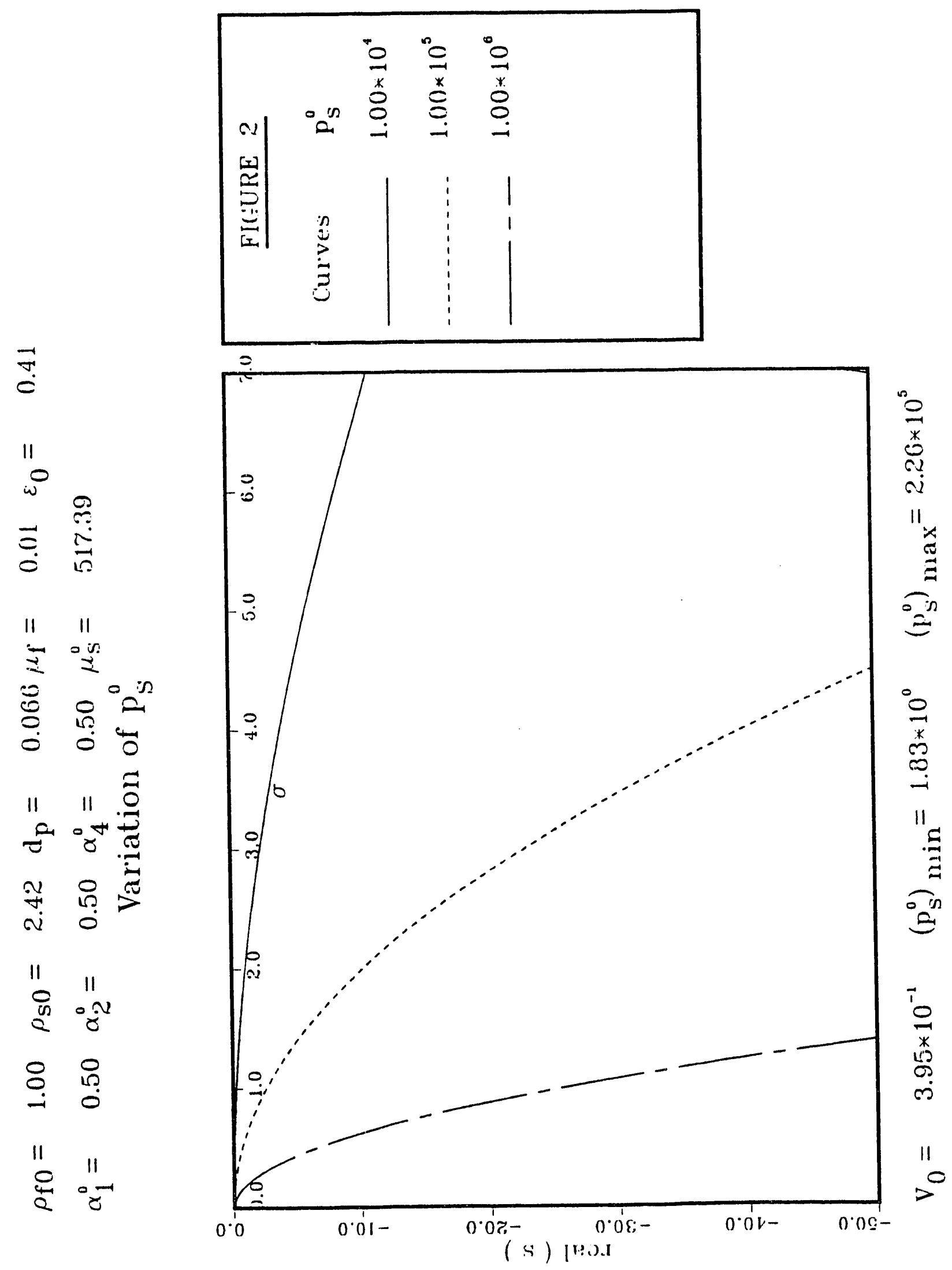




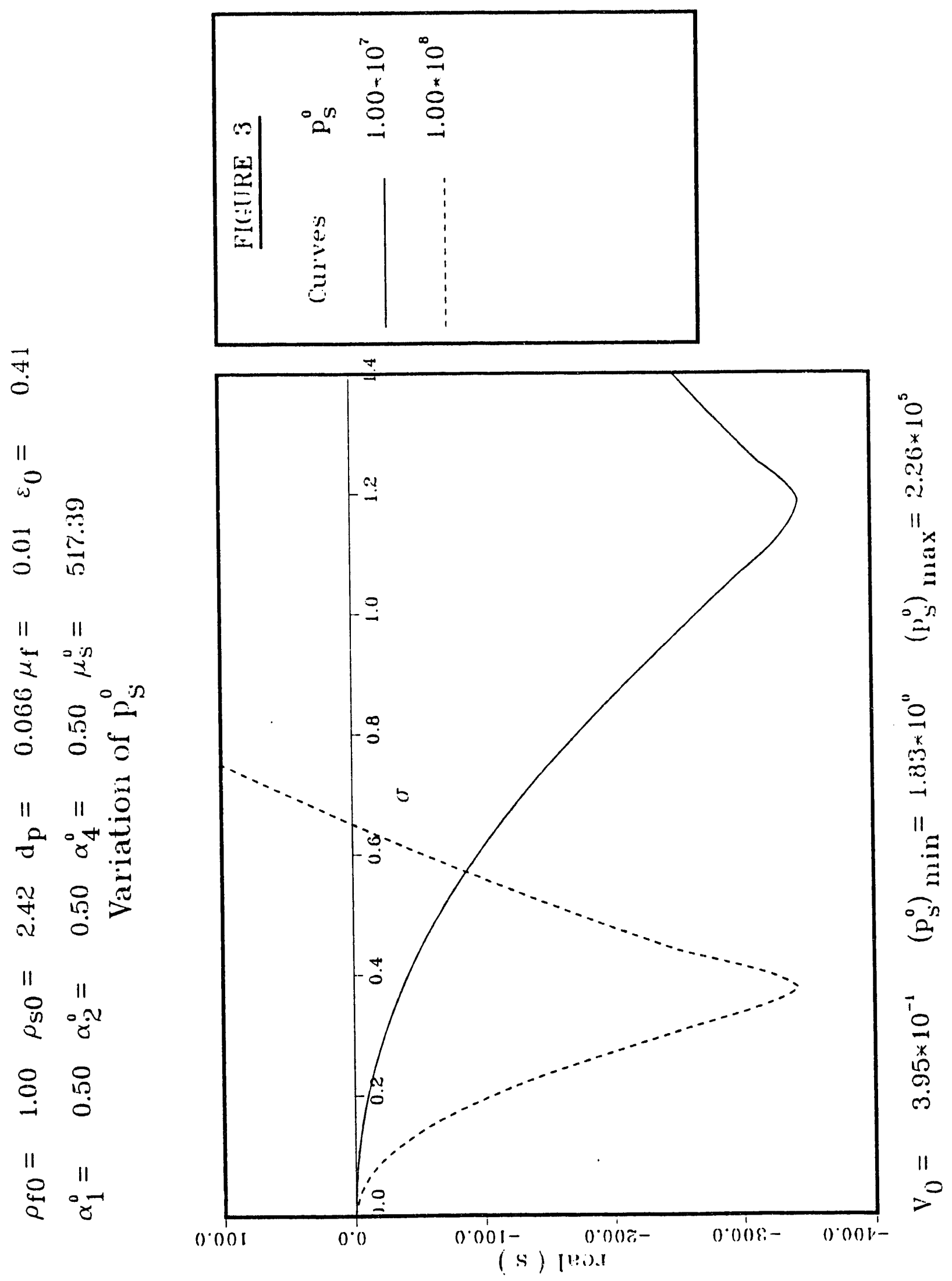




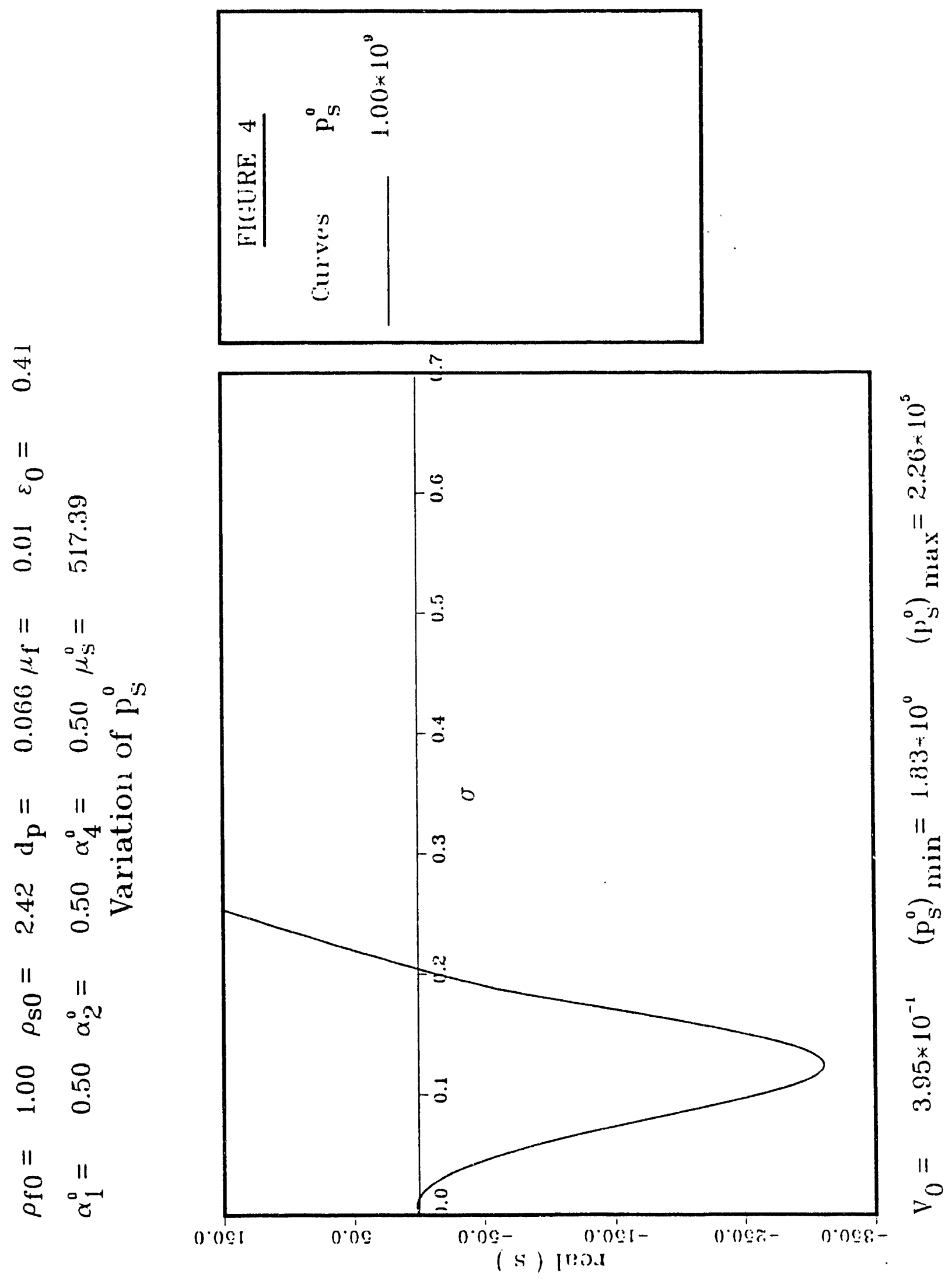




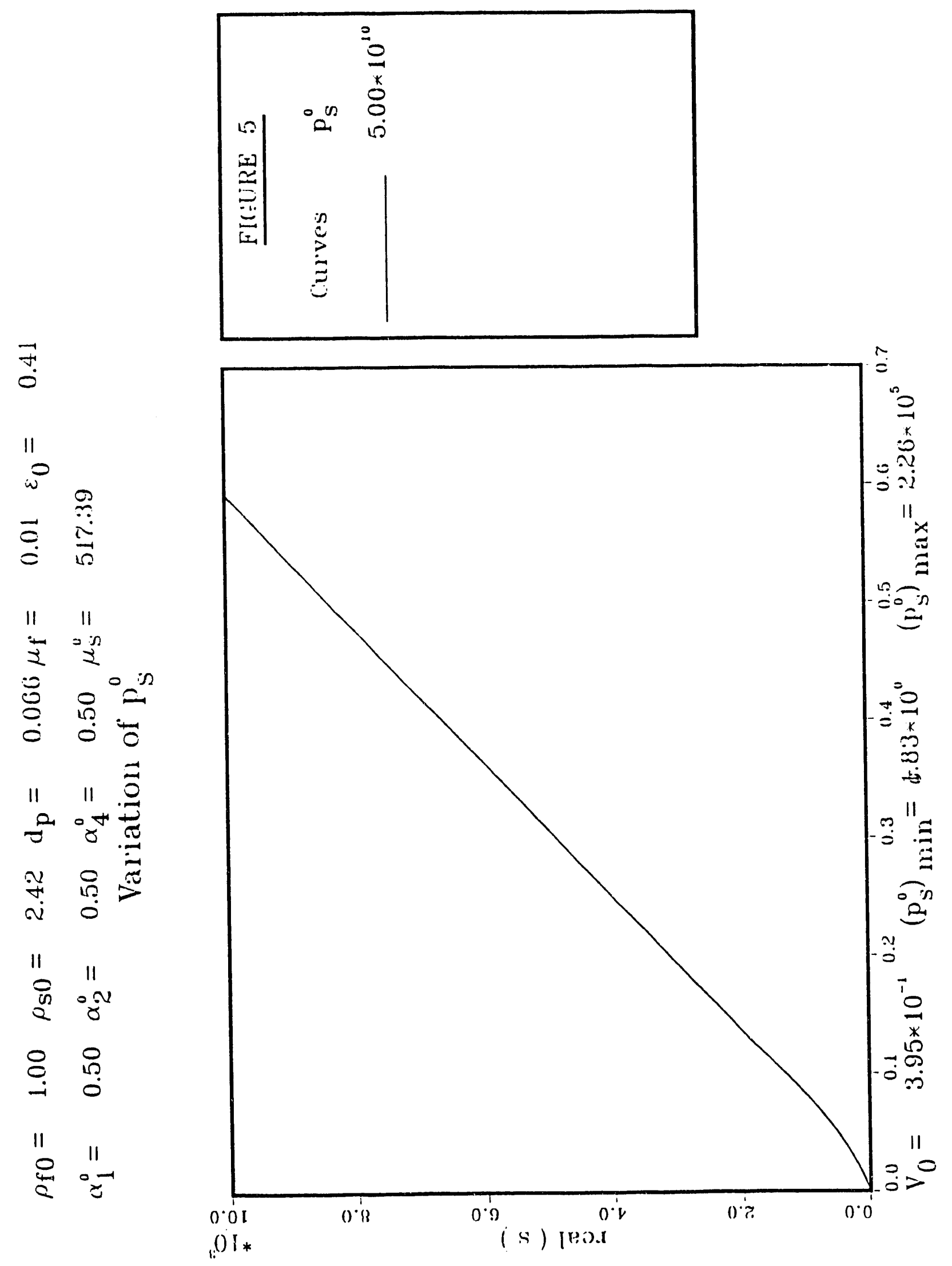




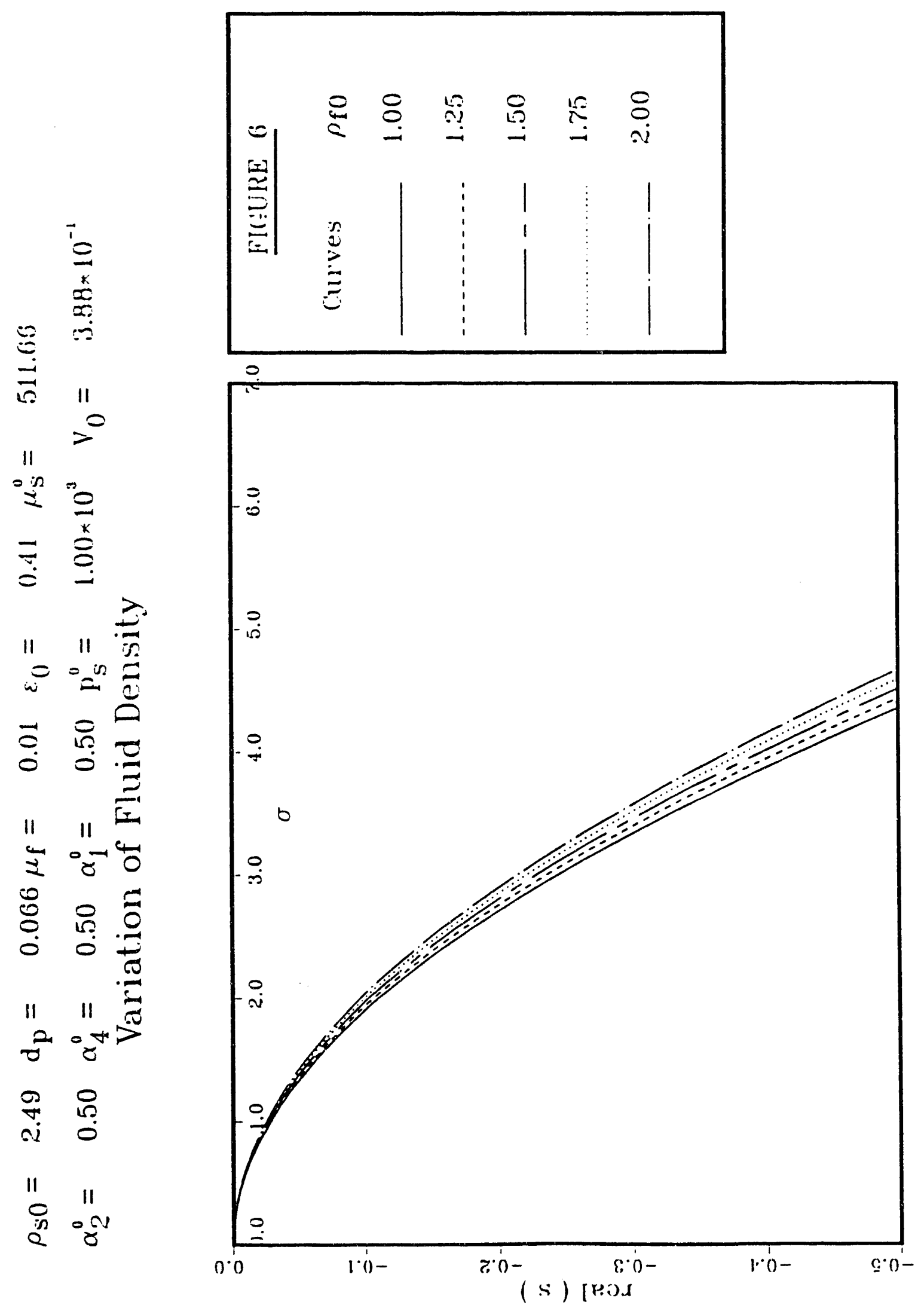




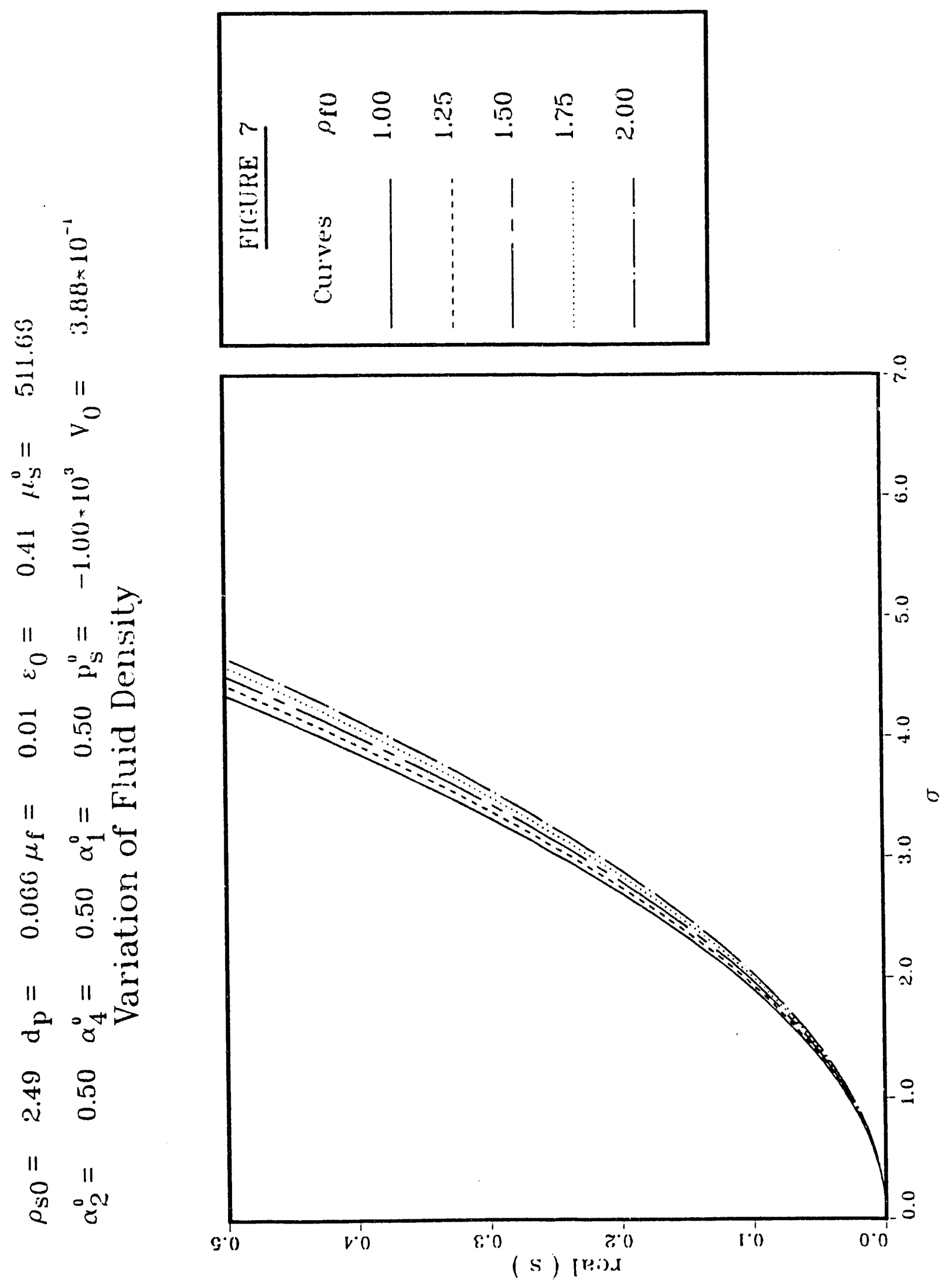




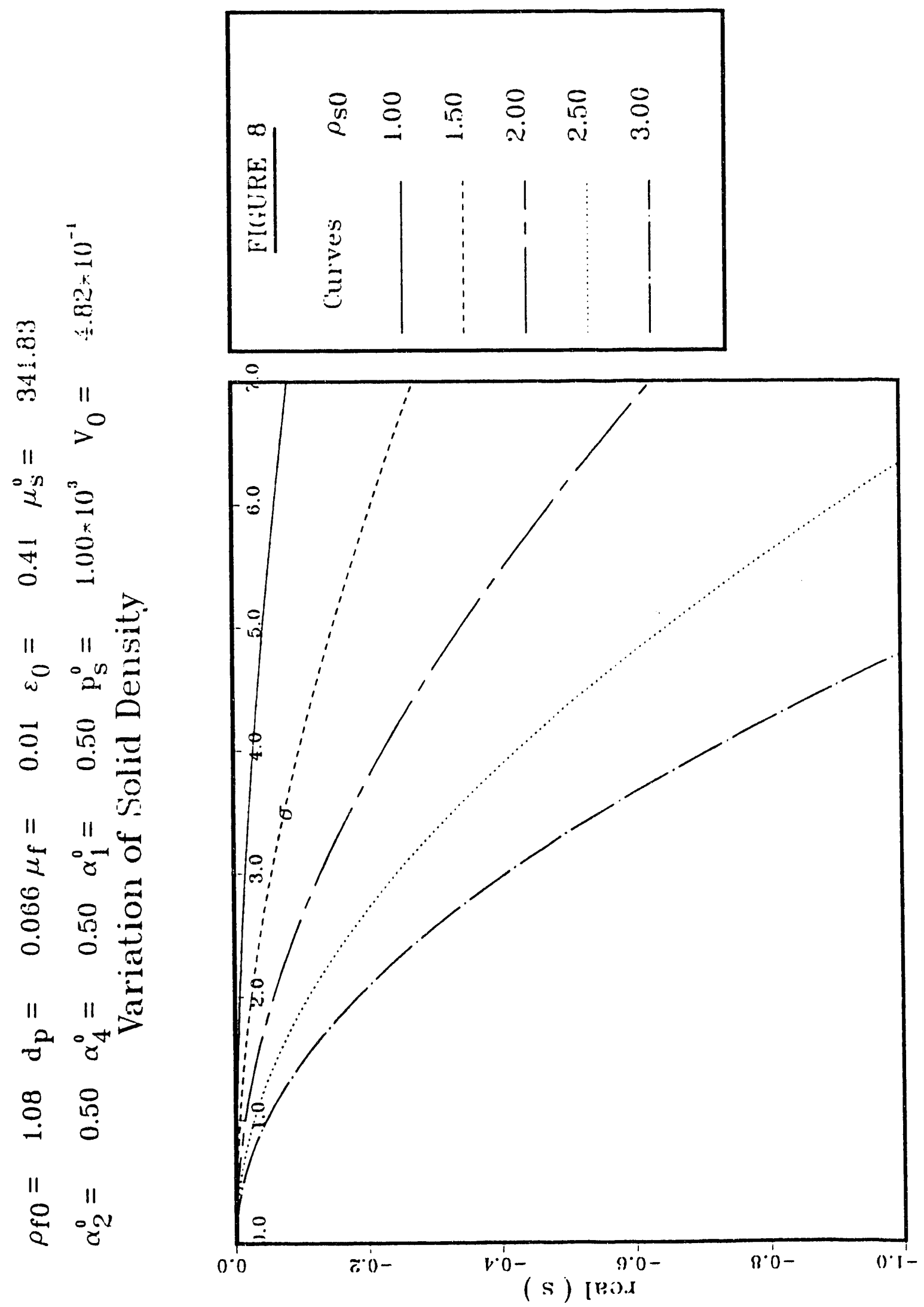




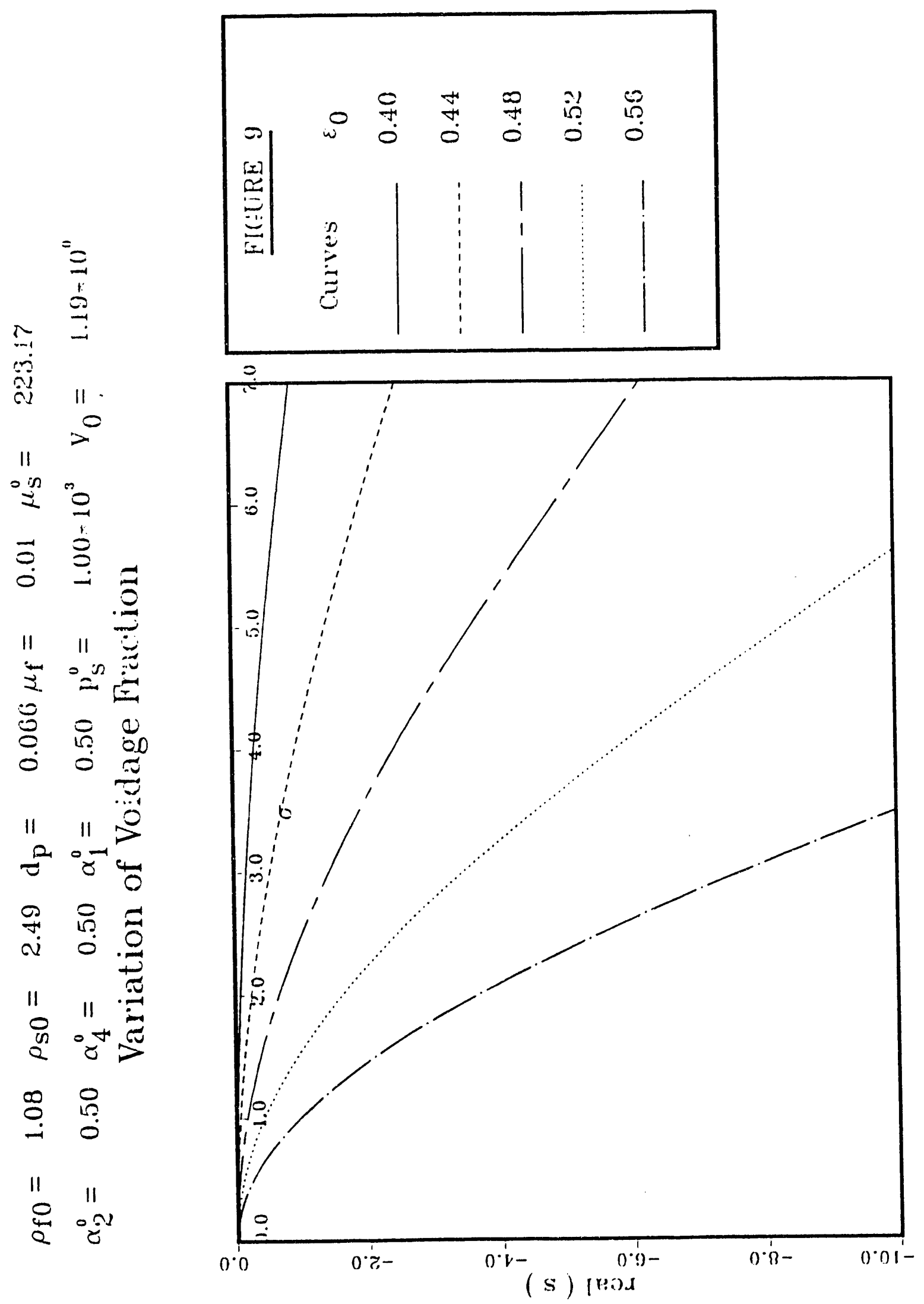




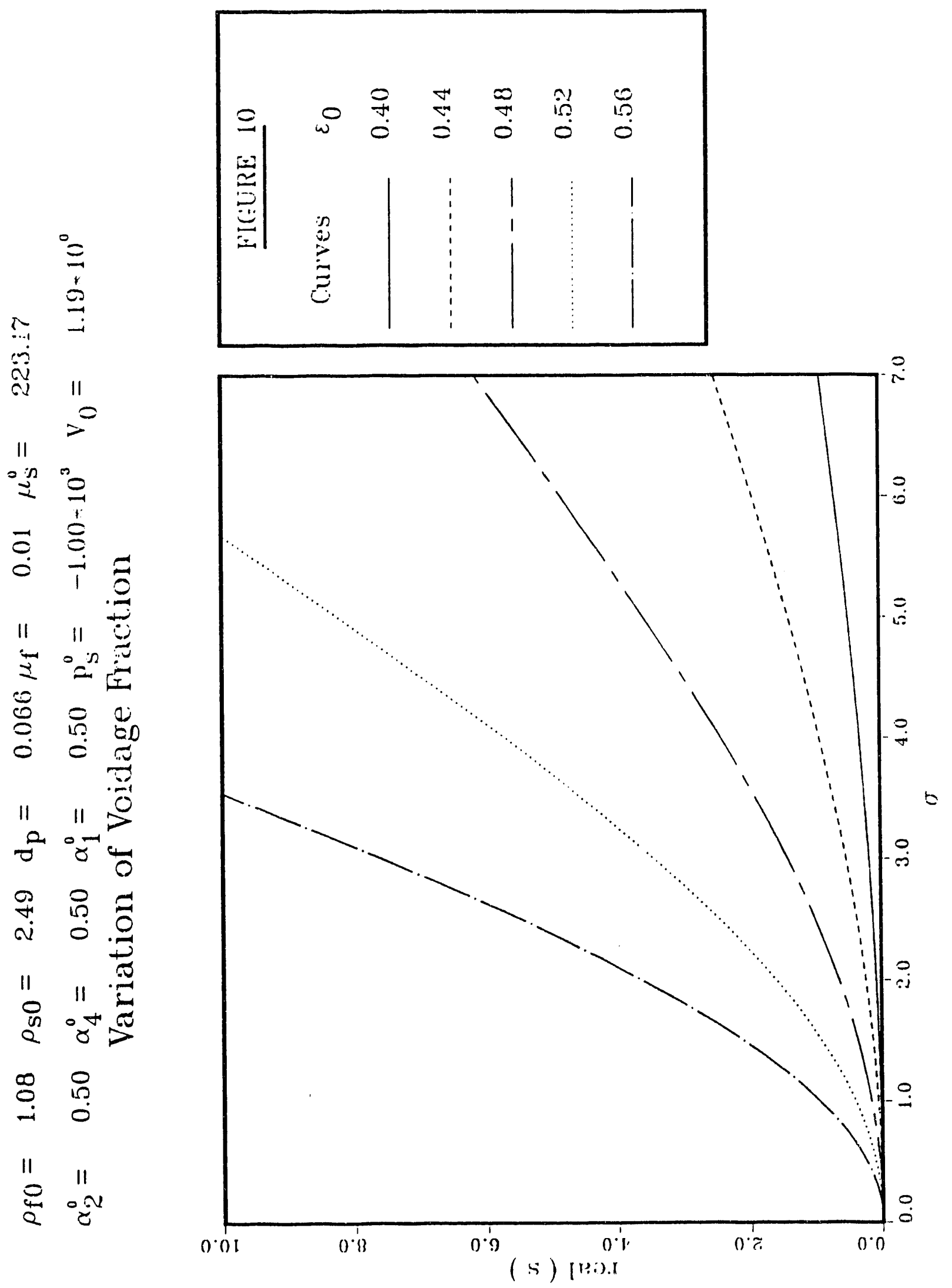




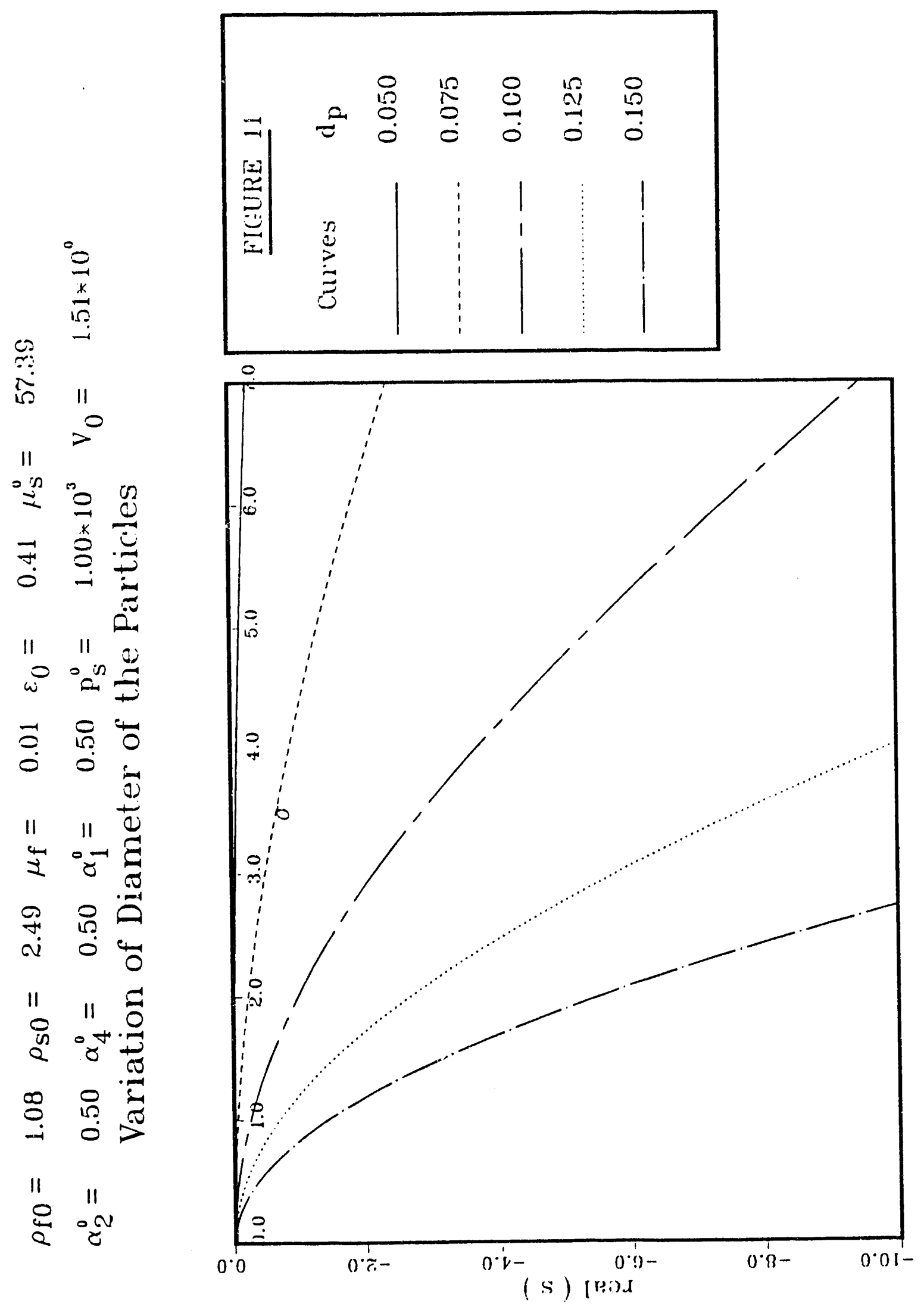




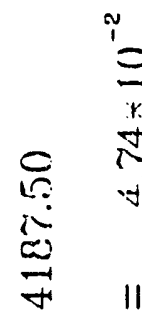

ב-

$11>$

$\stackrel{\circ}{2}$

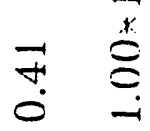

$\begin{array}{lll}11 & 11 & 0 \\ 0 & 0 & 0 \\ 0 & 0 & 0 \\ 0 & 0 & 0 \\ 0 & 0 & 0 \\ 0 & 0 & 0\end{array}$

1111 原

गี ช

궁

$\ln ^{\infty}$

$\begin{array}{ll}\infty & 0 \\ 0 & 0 \\ & 0\end{array}$

$\sum_{\substack{1 \\ 0}}^{11}$
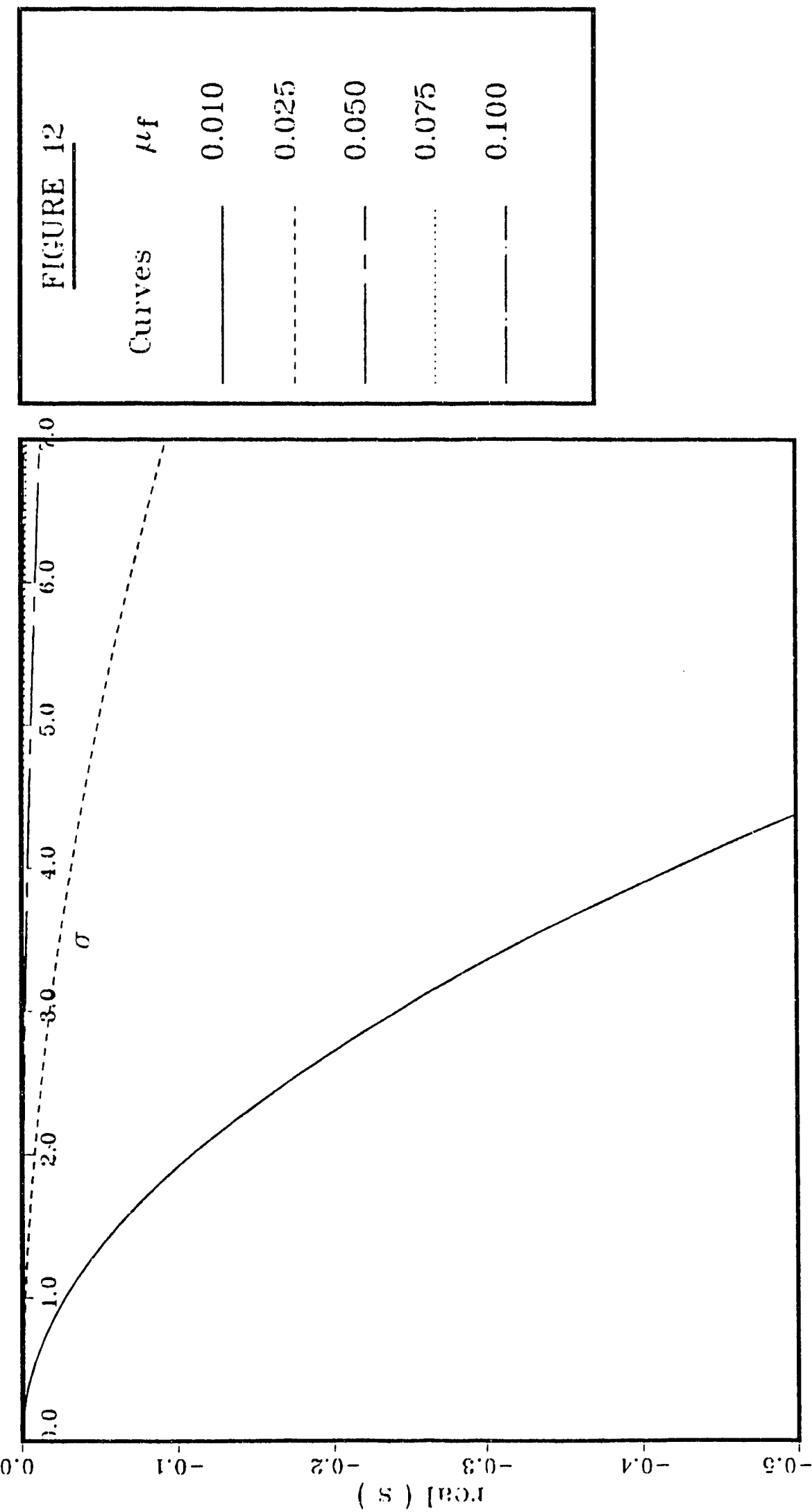


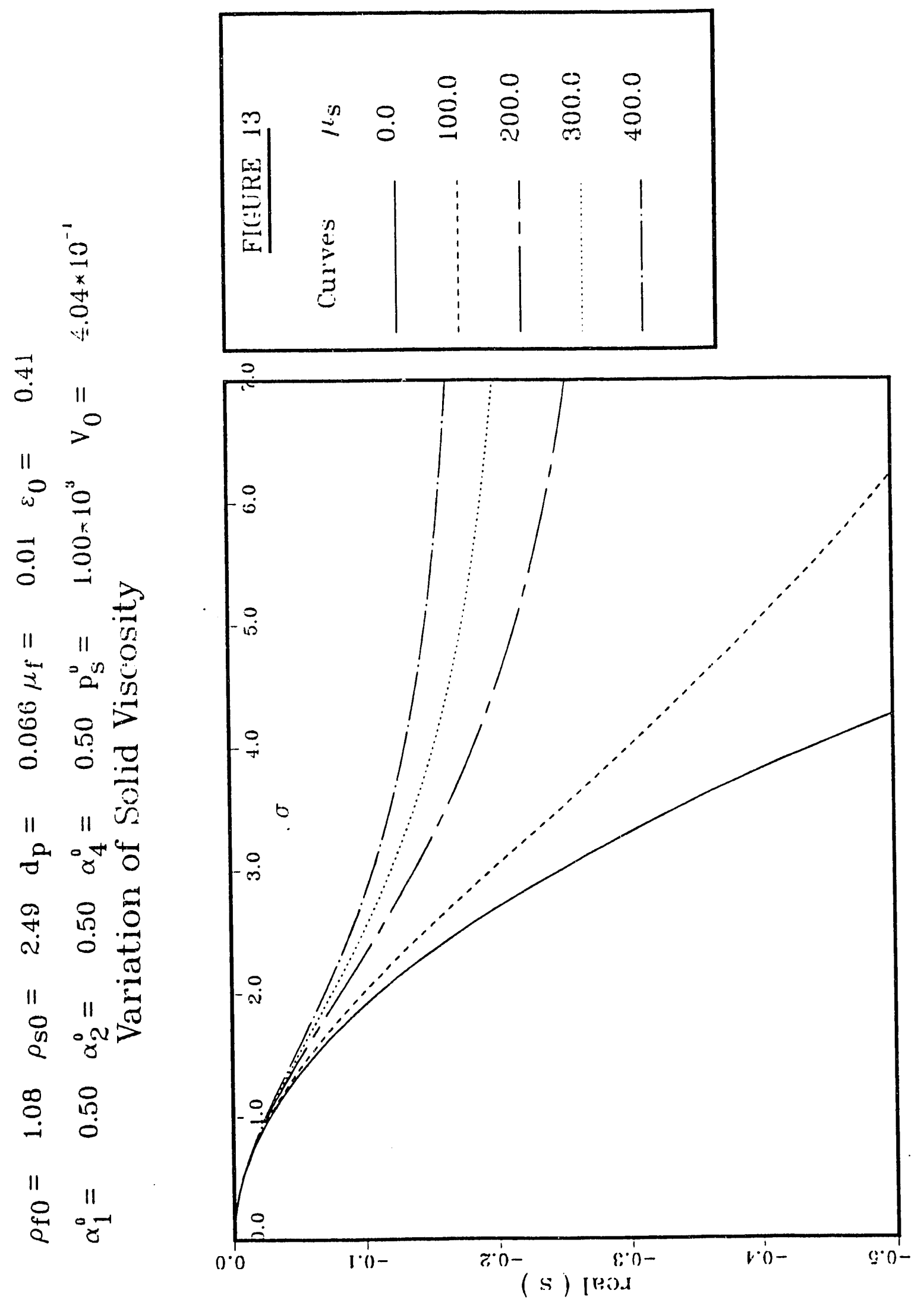




\section{APPENDIX A.}

\section{LITERATURE REVIEW}

A fluidized bed is a multicomponent system. In the past, attempts have been made to understand the phenomena of fluidization by modeling the fluidized bed based on the theory of multicomponent system. Two different techniques of mathematical modeling, namely averaging technique and mixture theory, are used to describe the behavior of the fluidized bed.

Theories of mixtures are motivated by the desire to obtain constitutive expressions, which are an homogenized representation of the micro structure of the constituents. The resulting averaged equations are far simpler to solve. The averaging procedures in the literature vary significantly. They are usually found to lead to equivalent forms of averaged balance equations.

Much of the early work in averaging theories stemmed from a desire to describe the behavior of a multiphase flow. Anderson and Jackson [ 1968 ], Jackson [1985 ], Drew and Segel [ 1971b ], Murray [ 1965 ], Pigford and Baron [ 1965 ] were among the first few researchers who adopted the averaging technique to multiphase flow. Anderson and Jackson [ 1968 ] modified point-wise equations of motion, valid for a single fluid or a single particle, to account for the presence of the other components and the interactions between the components. These equations were then averaged over some suitable volume which is large compared with some characteristic dimension ( e.g. mean particle spacing ), but small compared with the dimension of the whole system. Proper frameindifferent constitutive relations for these terms, as well as for the stress tensors for the each constituent, are then required. They assumed a fluid-like behavior for the solid constituent with the coefficient of viscosity depending upon the 
local mean voidage $\varepsilon=(1-v)$. The shortcoming of this assumption is discussed later. They also assumed that interaction term between the constituents is composed of the drag force ( proportional to the relative velocity) and the virtual mass force ( relative acceleration, proportional to the mass of the fluid displaced by the particle ). However, they did not derive an objective form for this term, rather they selected one of two different forms on the basis that the results are more sensitive for the form that they selected.

Drew and Segel [ 1971b] on the other hand, used a different more complex averaging technique and obtained equations of motion for a broad class of two phase flow conditions. Their averaging technique involves two integrations over spheres in three-space and two integrations in time for smoothing out discontinuities. They observed that a solution to the exact or instantaneous formulation could contain jumps or discontinuities. For example, the value of the density would jump when a constituent interface is crossed. The object of the averaging is to obtain a formulation whose solutions are smooth. If only one spatial averaging integration is used, the integrated or averaged density variable will be continuous, but their derivative can be discontinuous. Drew and Segel [ 1971b ] also pointed out certain inconsistencies in the derivations of Anderson and Jackson and Murray. By correcting these inconsistencies, Drew and Segel showed that various methods of derivation lead to similar balance equations for mass and momentum. However, in each case the terms appearing in these relations, such as averaged velocities and pressures, have interpretations which depend on the type of averaging employed. Drew and his coworkers also paid attention to the requirements of frame indifference in deriving their theories. Many of the expressions for virtual mass which had been used did not satisfy frame indifference.

Many researchers derive equations of motion by applying an averaging process to the microscopic equations. However, the choice of averaging process is 
dictated by the taste of the researcher as well as the particular problem studied. In complicated dispersed two phase flows, where the individual phase velocities are not equal nor the concentration of one of the phases small, volume averaging looses much of its appeal. As in the averaging of any set of non-linear field equations, the averaging process produces Reynolds stress like terms and interaction terms which ultimately must be modeled by some closure technique.

The alternative approach is to proceed axiomatically, using the techniques of continuum mechanics. This approach was first formulated by Truesdell [1965]. He formulated the interaction between several constituents by generalizing the equation and principles of the mechanics of a single continua. The fundamental assumption in this theory is that at any instant of time, every point in space is occupied by one particle from each constituent. A detailed treatment of the theory can be found in Müller [1968], Green and Naghdi [1969], Bowen [1976], Atkin and Craine [1976] and Bedford and Drumheller [1983]. Subsequently, many authors used this theory to study the behavior of fluidized beds. El-Kaissy [1975], [1976], Homsy et al. [1980] used this theory to propose a thermomechanical theory for fluidized bed. They adopted the mixture theory approach and derived constitutive relations that were properly invariant. An important part of his work was the emphasis placed upon the principle of material frame-indifference and the derivation of frame-indifferent (objective) combinations of the variables used in the constitutive equation. They formulated the linear instability wave problem and compared the result with the experimental work. However, the Cauchy's stress tensor for the solid constituent was assumed to be of the same form as that of fluid constituent.

Foscolo and Gibilaro [1984], [1985], [1987] in a series of paper have advocated the "particle bed model ", but such a model generalizes the idea of single particlefluid interaction. This could be useful when volume fraction is very low. However, in actual practice the volume fraction is usually between 0.4 and 0.8 
and, in that case, the single particle-fluid model looses much of its appeal and mixture or averaging theory proves to be more useful.

It is now clear that one of the main tasks is the modeling of the stress tensor for the solid constituent. In many of these studies the basic set of equations is not closed, even if all the material constants are specified. By that we mean, there are more unknowns than equations. The conservation of mass and linear momentum for the two constituents provide a system of eight equations from which the nine unknowns, u (velocity of the solid constituent), v (velocity of the fluid constituent $), v$ ( volume distribution function) and $\mathrm{p}_{\mathrm{f}}$ ( pressure of the fluid constituent ) and $\mathrm{p}_{\mathrm{s}}$ ( pressure of the solid constituent) need to be calculated. As mentioned earlier, there is much argument about the meaning of $\mathrm{p}_{\mathrm{s}}$. Anderson and Jackson [1968], and El-kaissy [1975] suggested a fluid-like behavior for the solid constituent. But, an inherent problem in assuming a fluid-like behavior for the solid constituent is defining the solid pressure term. The term $-\mathrm{p}_{f} \delta_{\mathrm{ij}}$ represents the constitutively indeterminate spherical stress in the fluid due to the constraint of incompressibility. No such analogy exists for the solid pressure $-\mathrm{p}_{\mathrm{s}}$ $\delta_{\mathrm{ij}}$. Some authors ( cf. Drew and Segel [1971a], [1971b], Homsy, et al., [1980] ) refer to the term $\left(p_{s}-p_{f}\right)$ as the "collisional pressure", which is assumed to be a strongly decreasing function of voidage. Murray [1965], [1967] explains that, based on experimental observation in fluidized beds, there is practically no noticeable noise and hence there is no physical explanation for this assumption. $\mathrm{He}$, therefore, neglected the effects of the collisions of the particles, which would give rise to a solid pressure term. Some researchers have suggested that the solid pressure term be neglected. Others have suggested setting $p_{s}=p_{f}$ but this is no more than an assumption and lacks any firm physical basis. This assumption is most likely to be valid in case of two phase flows where properties of both phases are not very much different, say water and steam. However, this is not true in the case of granular materials. Prosperetti and Jones [1987] have investigated the local flow in the vicinity of an isolated sphere, where their 
interpretations of the interface pressure account for the local inertia of the flow. By considering the flow of an inviscid fluid around a sphere, they have proposed the following form for the pressure difference:

$$
p_{s}-p_{f}=-\frac{1}{4} \gamma_{f}(\mathbf{v}-\mathbf{u})^{2}
$$

The interesting feature here is that the solid-phase pressure due to the presence of the particles is less than the bulk fluid pressure. Givler [ 1987 ] argues that such an assumption is contrary to physical interpretation. Finally, a fundamental shortcoming of assuming a fluid-like behavior is that, theoretically, in one limit, as the volume fraction $v$ approaches a maximum value, $v_{\mathrm{m}^{\prime}}$ the mixture of fluid and solid particles should behave as a granular material; in the other limit as $v$ approaches zero, the mixture should behave as a fluid. These limiting conditions, more specifically $v \rightarrow v_{m}$ are not described by assuming a fluid like behavior for the solid constituent. Indeed, the rheological behavior of granular materials is quite different from that of Newtonian fluids. For example, phenomena such as normal stress effects that are observed experimentally, cannot be predicted using a Newtonian model for the granular materials. Also in many cases it has been found that the mathematical model for the stress tensor for the solid constituent is not properly frame indifferent. This again raises questions about the validity of such a model.

Recently, Massoudi [ 1986 ], [ 1988 ] has developed a continuum theory for modeling fluidized beds in which he has described a properly frame indifferent mathematical model for the stress tensor for the solid constituent and this model also provides a closed set of equations. Massoudi used a proper frame indifferent term for the virtual mass term. The stress tensor for the mixture is formulated such that both the limits i.e., $v$ approaching zero and $v$ approaching $v_{m}$ (which is assumed to be unity, but could be any value ), are satisfied. Normal stress difference is one of the important phenomena observed in granular materials and can be predicted by the form for the stress tensor for the solid 
constituent which Massoudi has derived in his work. Our investigations will be based on this theory. 


\section{APPENDIX B.}

\section{DRAG CORRELATIONS}

It follows from equation (6-3) that

$$
\alpha_{3} V_{0}\left(\frac{\pi}{6} d_{p}^{3}\right)=\rho_{s} g v_{n}\left(\frac{\pi}{6} d_{p}^{3}\right)
$$

where $V_{0}$ is the velocity of the fluid at minimum fluidization. Once we know the minimum fluidization velocity and the volume fraction at minimum fluidization, we can obtain a value for $\alpha_{3}$ at those conditions from above equation (B-1).

There are number of methods available for calculating the minimum fluidization velocity. All of them are primarily derived from curve fitting the experimental results. We use a slightly modified version of Ergun's equation. In order to be consistent with our equations, where the drag force is balanced by the weight of the particle not the usual effective weight of the particle, we have to replace the term $\left(\rho_{\mathrm{s}}-\rho_{\mathrm{f}}\right)$ by $\rho_{\mathrm{s} 0} \phi_{\mathrm{s}^{\prime}}$ where $\phi_{\mathrm{s}}$ is the packing factor. The density of the solid particle, $\rho_{\mathrm{s} 0}$, is determined by the particle packing distribution. Reference density of the particle is calculated by the weight of the particle divided by the volume of the particle. But one can ask the question, which volume? Is it just the volume of the particles or, for example, the volume of the particles including the interstices. Again, the type of packing, size and distribution of particles also affects the density calculation. In an ideal case,we have monosized spheres and the type of packing we consider is an ordered packing. Then the maximum coordination number* ( $\mathrm{cf}$. German [ 1986 ]) is 12 and a fractional density of the

"Coordination number - The number of neighboring particles forming contacts with a given particle 
.7405 can be achieved. This type of packing is called face centered cubic packing. Generally, a packing coordination of 4 is required to achieve geometrical stability. A random packing assembled under the action of gravity requires each sphere that joins the packing rest on at least three contact points. Thus each sphere adds three new contacts to the packing. Since each contact point is shared by two spheres, the net increase in packing coordination number is six contacts per sphere. This implies that in the worst case, an average coordination number of 6 is expected for a stable packing of monosized spheres. The fractional density which corresponds to this case is $\frac{\pi}{6}$, which is the ratio of a volume of a sphere to that of a cube, the length of whose edge is the same as the diameter of the sphere. Any density which was calculated based on the particles being spherical has to be corrected by this factor. This fact is more pronounced in fluidized flows. Since in a fluidized bed when the weight of the particle is just supported by the upward flowing fluid, i.e., incipient fluidization, a coordination number of 6 is expected and any reference density which was calculated by an experimenter has to be corrected by this factor $\frac{\pi}{6}$. With this modification we use Ergun's equation for the calculation of minimum fluidization velocity.

\section{B.0.1 Modified Ergun's equation and calculation of minimum fluidization velocity}

Let us consider Ergun's equation ( cf. Geldart [ 1986 ])

$$
\frac{150\left(1-\varepsilon_{m f}\right)}{\varepsilon_{m f}^{3}} R e_{m f}+\frac{7 R e_{m f}^{2}}{4 \varepsilon_{m f}^{3}}=A r,
$$

where,

$$
R e_{m f}=\frac{\rho_{f} V_{0} d_{p}}{\mu},
$$




$$
A r=\frac{\rho_{f} d_{p}^{3} \rho_{s} g}{\mu^{2}}
$$

and

$$
\rho_{s}=\rho_{s e} \phi_{s}=\rho_{s e} \frac{\pi}{6}
$$

$\rho_{s e}$ is the density of the solid particle as reported by an experimenter.

Solving equation (B-2) for $\operatorname{Re}_{\mathrm{mf}}$ we have

$$
R e_{m f}=-\frac{300}{7} v+\sqrt{\frac{90000}{49} v^{2}+\frac{4 A r}{7}\left(1-v_{0}\right)^{3}} .
$$

From the above equation (B-2), we can calculate the minimum fluidization velocity, $\mathrm{V}_{0}$,

$$
V_{0}=\frac{R e_{m f} \mu}{d_{p} \rho_{f}}
$$

Once we calculate the minimum fluidization velocity $V_{0}$ from the equation $(B-4)$, we can calculate $\alpha_{3}$ from equation (B-1).

To check the validity of the theoretical expression for the minimum fluidization velocity for the range of parameters we are considering, we have compared it with the experimental results. We have taken the same set of numerical data as reported by Ham [ 1990 ] and the results are found to be within admissible error. The minimum and maximum errors were found to be around $0 \%$ and $17 \%$ respectively, which is even in the range of experimental errors. The detailed results are reported in a tabular form below. 
Table 1. Particle and Fluid Characteristics

\begin{tabular}{|c|c|c|c|c|c|c|}
\hline Sot & $\alpha_{p}$ & $\rho_{s e}$ & 1 & Fluid & $\mu$ & $\rho_{f}$ \\
\hline & $\mathrm{cm}$ & $\mathrm{g} / \mathrm{cm}^{3}$ & 1 & & poise & $\mathrm{g} / \mathrm{cm}^{3}$ \\
\hline A & 0.0655 & 2.42 & 1 & 1 & 0.0100 & 1.000 \\
\hline B & 0.0460 & 2.47 & 1 & 2 & 0.0175 & 1.041 \\
\hline C & 0.0325 & 2.49 & 1 & 3 & 0.0264 & 1.077 \\
\hline$D$ & 0.0230 & 2.47 & 1 & 4 & 0.0446 & 1.108 \\
\hline $\mathbf{E}$ & 0.0165 & 2.47 & 1 & 5 & 0.0974 & 1.146 \\
\hline
\end{tabular}

Table 2. Fluidized bed expansion characteristic

\begin{tabular}{|c|c|c|c|c|c|}
\hline \multirow{3}{*}{$\begin{array}{l}\text { Sot } \\
\text { A }\end{array}$} & \multirow{3}{*}{$\frac{\text { Fluid }}{1}$} & \multirow[b]{2}{*}{$\varepsilon_{\mathrm{mf}}$} & Expt1. & \multicolumn{2}{|l|}{ alculated } \\
\hline & & & $u_{m f}(\mathrm{~cm} / \mathrm{s})$ & $\operatorname{mf}(\mathrm{cm} / \mathrm{s})$ & \&Error \\
\hline & & $0.407 \pm 0.001$ & $0.450 \pm 0.050$ & 0.380 & +17.0 \\
\hline B & 1 & $0.409 \pm 0.002$ & $0.220 \pm 0.020$ & 0.203 & +8.00 \\
\hline$c$ & 1 & $0.410 \pm 0.020$ & $0.097 \pm 0.001$ & 0.104 & -7.00 \\
\hline D & 1 & $0.398 \pm 0.001$ & $0.052 \pm 0.060$ & 0.046 & +11.0 \\
\hline $\mathbf{E}$ & 1 & $0.411 \pm 0.001$ & $0.026 \pm 0.002$ & 0.027 & -4.00 \\
\hline c & 2 & $0.407 \pm 0.001$ & $0.059 \pm 0.006$ & 0.058 & +1.00 \\
\hline c & 3 & $0.410 \pm 0.001$ & $0.040 \pm 0.004$ & 0.040 & +0.00 \\
\hline c & 4 & $0.409 \pm 0.010$ & $0.026 \pm 0.003$ & 0.030 & +11.0 \\
\hline c & 5 & $0.413 \pm 0.010$ & $0.011 \pm 0.011$ & 0.011 & +0.00 \\
\hline
\end{tabular}




\section{BIBLIOGRAPHY}

1. Adkins, J. E., Non-linear Diffusion, 1. Diffusion and Flow of Mixture of Fluids, Phil. Trans. R. Soc. Lond.,Vol. A 255, 1963a, pp. 607.

2. Adkins, J. E., Non-linear Diffusion, 1. Constitutive Equations for Mixture of Isotropic Fluids, Phil. Trans. R. Soc. Lond. Vol. A 255, 1963b, pp. 635.

3. Agarwal, G. P., Hudson, J. L. and Jackson, R., Fluid Mechanical Description of Fluidized beds. Experimental Investigation of Convective Instabilities in Bounded Beds, Ind. Eng. Chem. Fundam., Vol. 19, 1980, pp. 59-65.

4. Ahmadi, G., A Generalized Continuum Theory for Granular Materials, Int. J. Non-linear Mechanics, Vol. 17, 1982, pp. 21-33.

5. Anderson, T. B. and Jackson, R., A Fluid Mechanical Description of Fluidized Beds, Ind. Eng. Chem. Fundamentals,Vol. 6, No. 4, 1967, pp. 527-539.

6. Anderson, T. B. and Jackson, R., Fluid Mechanical Description of Fluidized Beds: Stability of the State of Uniform Fluidization, Ind. Eng. Chem. Fundamentals, Vol. 7, No. 1, Feb. 1968, pp. 12-21.

7. Atkin, R. J. and Craine, R. E., Continuum Theories of Mixtures: Basic Theory and Historical Development, Quart. J. Mech. and Appl. Math.,Vol. 29, 1976, pp. 290.

8. Ayazi Shamlou, P., Liu, J. and Yates, J. G., Hydrodynamic Influences on Particle Breakage in Fluidized Beds, Chem. Engg. Sci.,Vol. 45, No. 4, 1990, pp. 809-817.

9. Batchelor, G. K., A New Theory of the Instability of a Uniform Fluidized Bed, I. Fluid Mechanics, Vol. 193, 1988, pp. 75-110.

10. Bedford, A. and Drumheller, D. S., Recent Advances, Theories of Immiscible and Structured Mixtures, Int. J. Engg. Sc.,Vol. 21, 1983, pp. 863.

11. Botterill, J. S. M., Fluid-Bed Heat Transfer, Academic Press, 1975.

12. Bowen, R. M., Theory of Mixtures, Continuum Physics, Vol. 3, 1976, pp. 1. 
13. Cowin, S. C., A Theory for the flow of Granular Materials, Powder technology, Vol. 9, 1974a, pp. 61-69.

14. Cowin S. C., Constitutive Relations that Imply a Generalized MohrCoulomb Crite, Acta Mechanica,Vol. 20, 1974b, pp. 41-46.

15. Carey, V. P., Dependence of Settling Velocity on Particle Concentration in a Fluidized Bed of Spherical Particles, Int. J. Multiphase Flow, Vol. 13, No. 3, 1987, pp. 429-431.

16. Chandrasekhar, S., Hydrodynamic and Hydromagnetic Stability, Dover, New York, 1981.

17. Davidson, J. F., Clift, R. and Harrison, D., Fluidization, Academic Press, London and New York, 1985.

18. Davidson, J. F. and Harrison, D., editors, Drying in Fluidization, Academic Press, London and New York, 1971.

19. Drazin, P. G. and Reid, W. H., Hydrodynamic Stability, Cambridge University Press, Cambridge, 1982.

20. Drew, D. A., Averaged Field Equations for Two Phase Media, Studies in Applied Mathematics,Vol. L, No. 2, 1971a, pp. 133-166.

21. Drew, D. A. and Segel, L. A., Averaged Equations for Two Phase Flows, Studies in Applied Mathematics,Vol. L, No. 3, 1971b, pp. 205-257.

22. Drew, D. A., Lift-generated Instability of the Plane Couette Flow of a fluid, The Physics of Fluids, Vol. 18, No. 8, August 1975, pp. 935-938.

23. Drew, D. A., Effect of the Lift Force on the Stability of Uniform Fluidization, The Physics of Fluids,Vol. 19, No. 11, Nov. 1976, pp. 1716-1720.

24. Drew, D. A., Cheng, L. and Lahey Jr., R. T., The Analysis of Virtual Mass Effects in Two-Phase flow, Int. J. Multiphase Flow,Vol. 5, 1979, pp. 233.

25. Drew, D. A. and Lahey Jr., R. T., The Virtual Mass and Lift Force on a Sphere in Rotating and Straining Inviscid Flow, Int. I. Multiphase Flow, Vol. 13, No. 1, 1987, pp. 113-121.

26. El-Kaissy, M. M., The Thermomechanics of Multiphase Systems with Application to Fluidized Continua, Ph. D. Thesis, Stanford University, Stanford CA., 1975. 
27. El-Kaissy, M. M. and Homsy, G. M., Instability Waves and the Origin of Bubbles in Fluidized Beds-I. Experiments, Int. J. of Multiphase Flow,Vol. 2, 1976, pp. 379-395.

28. Ergun, S., Fluid Flow Through Packed Columns, Chemical Engineering Progress, Vol. 48, No. 2, Feb. 1952, pp. 89-94.

29. Flood, H. W. and Lee, B. S., Fluidization, Scientific American,Vol. 219, 1968, pp. 94.

30. Foscolo, P. U. and Gibilaro, L. G., A Fully Predicative Criterion for the Transition Between Particulate and Aggregate Fluidization, Chem. Engg. Sci.,Vol. 39, No. 12, 1984, pp. 1667-1675.

31. Foscolo, P. U. and Gibilaro, L. G., Fluid Dynamic Stability of Fluidized Suspensions: The Particle Bed, Chem. Engg. Sci.,Vol. 42, No. 6, 1987, pp. 1489-1500.

32. Foscolo, P. U., Gibilaro, L. G., Di Felice, R. and Waldram, S. P., The effect of interparticle forces on the stability of fluidized beds, Chem. Engg. Sci. Vol. 40, No. 12, 1985, pp. 2379-2381.

33. Foscolo, P. U., Di Felice, R., Gibilaro, L. G., Pistone, L. and Piccolo, V., Scaling relationships for fluidization: the generalised bed model, Chem. Engg. Sci.,Vol. 45, No. 6, 1990, pp. 1647-1651.

34. Ganser, G. H. and Drew, D. A., Nonlinear stability analysis of a uniformly fluidized bed, Int. J. Multiphase Flow, Vol. 16, No. 3, 1990, pp. 447-460.

35. Garg, S. K. and Pritchett, J. W., Dynamics of Gas-fluidized Beds, L. Appl. Phys.,Vol. 46, No. 10, 1975, pp. 4493-4500.

36. Geldart, D., Gas Fluidization Technology, John Wiley \& Sons, New York, 1986.

37. German, Randall M., Particle Packing Characterisitics, Metal Powder Industries Federation, Princeton NJ, 1986.

38. Gibilaro, L. G., Di Felice, R. and Waldram, S. P., Generalized friction factor and drag coefficient correlations for fluid-particle interactions, Chemicai Engineering Science, Vol. 40, No. 10, 1985, pp. 1817-1823.

39. Gibilaro, L. G., Di Felice, R. and Foscolo, P. U., The influence of Gravity on the Stability of Fluidized Beds, Chemical Engg. Science,Vol. 41, 1986, pp. 2438-2440. 
40. Gibilaro, L. G., Di Felice, R. and Foscolo, P. U., Added Mass Effects in Fluidized Beds: Application of the Geurst - Wallis Analysis of Inertial Coupling in Two Phase Flow, Chemical Engg. Science, Vol. 45, No. 6, 1990, pp. 1561-1565.

41. Gibilaro, L. G., Foscolo, P. U., Waldram, S. P., Felice, R. Di. and Hossain, I., A Review of Applications of a Fluid-Particle Interaction model to Predictions of Fluidized Bed Behavior, Chemical Eng. Comm.,Vol. 62, 1987, pp. 17-29.

42. Gidaspow, D. and Ettehadieh, B., Fluidization in Two Dimensional Beds with a jet 2. Hydrodynamic Stability, Ind. Eng. Chem. Fund.,Vol. 22, 1983, pp. 193-201.

43. Givler, R. C., An Interpretation for the Solid-Phase Pressure in Slow, Fluid-Particle Flows, Int. J. Multiphase Flows,Vol. 13, No. 5, 1987, pp. 717-722.

44. Goodman, M. A. and Cowin, S. C., A continuum theory for Granular Materials, Arch. Rational Mechanics Anal.,Vol. 44, 1971, pp. 249-266.

45. Grace, J. R. and Tout, J. , A Theory for Cluster Formation in Vertically Converged Suspensions of Intermediate Density, Institution of Chemical Engineers, Vol. 57, 1979, pp. 49-54.

46. Green, A. E. and Naghdi, P. M., On Basic Equations for Mixtures, Quart. J. Mech. Appl. Math.,Vol. 22, 1969, pp. 427-438.

47. Green, D. and Homsy, G. M., Instabilities in Self-Fluidized Beds-I, Int. I. Multiphase flow, Vol. 13, No. 4, 1987a, pp. 443-458.

48. Green, D. and Homsy, G. M., Instabilities in Self-Fluidized Beds-II Experimen!s, Int. I. Multiphase Flows, Vol. 13, No. 4, 1987b, pp. 459-475.

49. Ham, J. M., Thomas, S., Guazzelli, E., Homsy, G. M. and Anselmet, M. -C., An Experimental Study of the Stability of Liquid - Fluidized Beds, Intl. I. Multiphase Flow, Vol. 16, No. 2, 1990, pp. 171-185.

50. Homsy, G. M., El-Kaissy, M. M. and Didwania, A., Instability Waves and the Origin of Bubbles in Fluidized Beds-II, Comparison with Theory, Int. I. Multiphase Flows, Vol. 6, 1980, pp. 305-318.

51. Howard, J. R., Fluidized Beds: Combustion and Applications, Applied Science Publishers, London and New York, 1983.

52. Howard, J. R., Fluidized Bed Technology, Principles and Applications, Adam Higler, Bristol and New York, 1989. 
53. Ishii, M., Thermo-Fluid Dynamic Theory of Two Phase Flow., Eyrolles, Paris, 1975.

54. Jackson, R., The Mechanics of Fluidized Beds: Part I: The Stability of the State of Uniform Fluidization, Trans. Instn. Chem. Engrs.,Vol. 41, 1963, pp. 13-21.

55. Jackson, R., The Present Status of Fluid Mechanical Theories of Fluidization, Chem. Engg. Prog. Symp. Series, Vol. 66, 1970, pp. 3.

56. Johnson, G., Massoudi, M. and Rajagopal, K. R., A review of interaction mechanisms in Fluid-Solid flows, Technical Report, U.S. Dept. of Energy,DOE/PETC/TR-90/9, 1990.

57. Johnson, G., Ph. D. Thesis, University of Pittsburgh, Pittsburgh, PA, 1991a.

58. Johnson, G., Massoudi, M., and Rajagopal, K. R., Flow of a fluid infused with solid particles through a pipe, Int. J. Engg. Science.,Vol. 29, No. 6, 1991b, pp. 649-661.

59. Johnson, G., Massoudi, M., and Rajagopal, K. R., Flow of a fluid-solid mixtures between flat plates, Chemical Engg. Science.,Vol. 46, No. 7, 1991c, pp. 1713-1723.

60. Khan, A. R. and Richardson, J. F., Fluid-Particle Interactions and Flow Characteristics of Fluidized Beds and Settling Suspensions of Spherical Particles, Chem. Engg. Comm.,Vol. 78, 1989, pp. 111-130.

61. Knops, R. J. and Wilkes, E. W., Elastic Stability, Flügge's Handbuch der Physik, ed. C. Truesdell, Volume $=\{\mathrm{VIa} / 3$, Berlin, Springer-Verlag, 1973.

62. Kunii, D. and Levemspiel, O., Fluidization Engineering, Krieger Publishing Co., Florida, 1984.

63. Lin, C. C., The Theory of Hydrodynamic Stability, Cambridge University Press, Cambridge, 1955.

64. Liu, J. T. C., Note on a Wave-hierarchy Interpretation of Fluidized Bed Instabilities, Proc. R. Soc. Lond., Vol. A 380, 1982, pp. 229-239.

65. Liu, J. T. C., Nonlinear Unstable Wave Disturbances in Fluidized Beds, Proc. R. Soc. Lond.,Vol. A 389, 1983, pp. 331-347.

66. Massoudi, M., Ph. D. Thesis, University of Pittsburgh, Pittsburgh PA, 1986. 
67. Massoudi, M. and Boyle, E. J., Continuum Theories of Granular Materials with Applications to Fluidized Beds, Technical Note, DOE/METC-88/4077, May, 1987.

68. Massoudi, M., Stability Analysis of Fluidized Beds, Int. I. Engg. Sci.Vol. 26, 1988, pp. 765-769.

69. Massoudi, M., Rajagopal, K. R., and Ekmann, J. M, On the Viscosity of a Mixture of Particulate Solids and a Fluid Continuum, Topical Report, DOE/PETC/TR-90/8, Sept. 1990.

70. Massoudi, M. and Boyle, E. J., A review of Theories for Flowing Granular Materials with Applications to Fluidized Beds and Solid Transport, Topical Report, DOE/PETC/TR-91/8, May, 1991.

71. Medlin, J., Wong, H. and Jackson, R., Fluid Mechanical Description of Fluidized Beds. Convective Instabilities in Bounded Beds, Ind. Eng. Chem. Fundam. Vol. 13, No. 3, 1974, pp. 247-259.

72. Molerus, O., Hydrodynamic Stability of the Fluidized Bed, Proceedings of the International Symposium on Fluidization, 1967.

73. Müller, I., A Thermodynamic Theory of Mixture of Fluids, Arch. Ration. Mech. Anal.,Vol. 28, 1968, pp. 1-39.

74. Murray, J., On the Mathematics of Fluidization-I., Journal of Fluid Mechanics,Vol. 21, 1965, pp. 465-493.

75. Murray, J., On the Viscosity of a Fluidized System., Rheol. Acta,Vol. 6, 1967, pp. 27-30.

76. Mutsers, S. M. P. and Rietema, K., The Effect of Interparticle Forces on the Expansion of a Homogeneous Gas-Fluidized Bed, Powder Technology,Vol. 18, 1977, pp. 239-248.

77. Needham, D. J. and Merkin, J. H., The Propagation of a Voidage Disturbance in a Uniformly Fluidized Bed, I. Flu. Mech.,Vol. 131, 1983, pp. 427-454.

78. Orr, Clyde, Jr., Particulate Technology, The Macmillan Company, New York, 1966.

79. Passman, S. L. and Nunziato, J. W., Flow of a fluid saturated granular material, Mechanics of Structured Continua, Part A, 1981, pp. 343-353.

80. Passman, S. L. Nunziato, J., W. and Walsh, E. K.,, $A$ Theory of Multiphase Mixtures, Sandia report, SAND 82-2261, 1983. 
81. Passman, S. L., Nunziato, J. W., Bailey, P. B. and Reed, K. W., Shearing motion of a fluid-saturated granular material, I. Rheology,Vol. 30, 1986, pp. 167.

82. Passman, S. L. and Drew, D. A., Exact solutions in a theory of multicomponent mixtures., To appear, 1990.

83. Pigford, R. L. and Baron, T., Hydrodynamic Stability of a Fluidized Bed, Ind. Engg. Chem. Fund.,Vol. 4, 1965, pp. 81.

84. Prosperetti, A. and Jones, A. V., Pressure Forces in Disperse two Phase Flow, Int. J. Multiphase Flow, Vol. 10, 1984, pp. 425-440.

85. Prosperetti, A. and Jones, A. V., The Linear Stability of General TwoPhase Flow Models-II, Int. J. Multiphase Flow,Vol. 13, No. 2, 1987, pp. 161-171.

86. Rajagopal, K. R., and Massoudi, M., A Method for Measuring the Material Moduli of Granular Materials: Flow in Orthogonal Rheometer, Topical Report DOE/PETC/TR-90/3, Jan. 1990a.

87. Rajagopal, K. R., Massoudi, M. and Ekmann, J. M., Mathematical modeling of fluid-solid mixtures, In Recent Developments in Structured Continua, Editors D. De Kee and P. N. Kaloni, Longman, London, Vol. II, $1990 \mathrm{~b}$.

88. Rietema, K. and Piepers, H. W., The Effect of Interparticle Forces on the Stability of Gas-Fluidized Beds - I. Experimental Evidence, Chem. Engg. Science,Vol. 45, No. 6, 1990, pp. 1627-1639.

89. Rowe, P. N., The effect of pressure on minimum fluidization velocity, Chem. Engg. Sci.,Vol. 39, No. 1, 1984, pp. 173-174.

90. Ruckenstein, E. and Tzeculescu, M., On the hydrodynamics of the Fluidized Bed, Proceedings of the International Symposium on Fluidization, 1967.

91. Savage, S. B., Gravity Flow of Cohesion-less Granular Materials in Chutes and Channels, I. flu. Mech.,Vol. 92, 1979, pp. 53-96.

92. Savage, S. B., The Mechanics of Rapid Granular Flows, Adv. applied flows, Vol. 24, 1984.

93. Sommerfield, A, Ein Beitrag Zur Hydrodynamischen Erklaerung Der Turbulenten Fluessigkeitsbewegungen, Proceedings 4th International Congress of Mathematicians, Rome, Vol. III, 1908, pp. 116-124. 
94. Truesdell, C., Sulle Basi Della Thermomeccanica, Rand. Lincei.,Vol. 22, No. 8, 1957, pp. 33-38 and 158-166.

95. Truesdell, C. and Noll, W., The Non-Linear Field Theories of Mechanics, Handbuch der Physik (ed. Flugge, Springer),Vol. III/3, 1965.

96. Truesdell, C., Rational Thermodynamics, Springer, Berlin, 1984.

97. Walton, O. R. and Braun, R. L., , Journal of Rheology, Vol. 30, 1986, pp. 949.

98. Walton O. R. and Braun, R. L., , Acta Meccanica,Vol. 63, 1986, pp. 73. 

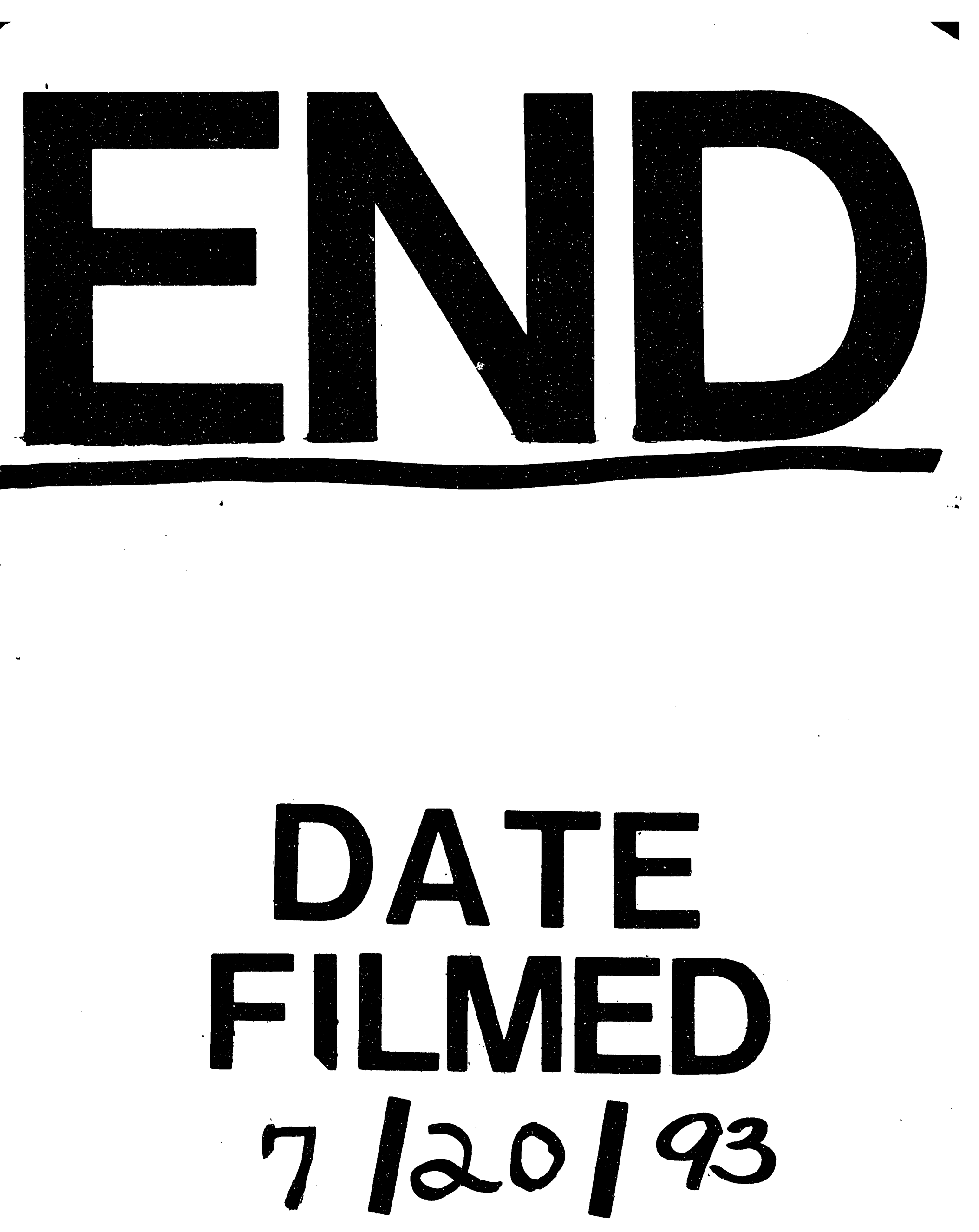
